\title{
Flow behaviour and aeroacoustic characteristics of a simplified high-speed train bogie
}

\author{
J. Y. Zhu' ${ }^{1}$, Z. W. Hu ${ }^{1}$, D. J. Thompson ${ }^{2}$ \\ ${ }^{1}$ Aerodynamics and Flight Mechanics Research Group, Faculty of Engineering and the Environment, \\ University of Southampton, Southampton SO17 1BJ, United Kingdom \\ ${ }^{2}$ Institute of Sound and Vibration Research, Faculty of Engineering and the Environment, University of \\ Southampton, Southampton SO17 1BJ, United Kingdom
}

\begin{abstract}
Aerodynamic noise becomes significant for high-speed trains and its prediction in an industrial context is difficult to achieve. The aerodynamic and aeroacoustic behaviour of the flow past a simplified high-speed train bogie at scale 1:10 is studied using a two-stage hybrid method comprising computational fluid dynamics and acoustic analogy. The near-field unsteady flow is obtained by solving the Navier-Stokes equations numerically with the delayed detachededdy model and the results are used to predict the far-field noise through the Ffowcs WilliamsHawkings method. The sound radiated from the same scaled bogie model is measured in an anechoic open-jet wind tunnel. The aeroacoustic characteristics of tandem wheelsets are also investigated for comparison. It is found that the unsteady flow past the bogie is characterized by coherently alternating vortex shedding from the axles and more randomly distributed vortices of various scales and orientations from the wheels and frame. The vortices formed behind the upstream geometries are convected downstream and impinge on the downstream bodies, generating a highly turbulent wake behind the bogie. The noise predictions correspond fairly well with the experimental measurements for the dominant frequency of tonal noise and the shape of spectra. Vortex shedding from the axles generates the tonal noise with the dominant peak corresponding to the vortex shedding frequency. The directivity exhibits a dipole shape for the noise radiated from the bogie. Compared to the wheelsets of the bogie, the noise contribution from the bogie frame is relatively weaker.
\end{abstract}

\section{Introduction}

Over the last few decades, researches have been conducted regarding the source mechanisms of flow-induced noise, particularly in aerospace engineering for landing gears and airframes [1,2]. Normally, a certain level of simplification was applied to the real geometry to focus on the dominant flow physics and the main noise mechanisms.

E-mail addresses:

j.zhu@soton.ac.uk (J.Y.Zhu), z.hu@soton.ac.uk (Z.W. Hu), djt@isvr.soton.ac.uk (D. J. Thompson) 
Being representative of a simplified geometry for many practical applications, the cylinders in tandem formation have been used to investigate the flow interaction and noise generation mechanisms of bluff bodies. Recently, as a Benchmark problem for Airframe Noise Computations (BANC-I \& BANC-II), the flow behaviour and noise radiation from tandem cylinders have been measured and the databases are used to assess the accuracy of different numerical methods for aeroacoustic applications $[3,4]$.

It is generally accepted that the aerodynamic noise becomes predominant for highspeed trains running at speeds over about $300 \mathrm{~km} / \mathrm{h}[5,6]$. Much progress has been made in the understanding of the aerodynamic phenomena associated with high-speed trains [7]. In contrast, the generation of aerodynamic noise from high-speed trains is less well understood. Most aeroacoustic studies have been performed through wind tunnel and track-side experiments [8,9]. Numerical calculations have been restricted to some simple geometries. The aeroacoustic calculations were performed on a forwardbackward facing step pair to simulate the pantograph cavity on the roof of a high-speed train [6]. The aerodynamic and aeroacoustic behaviour of the flow past an isolated wheelset, the main component of a train bogie, was studied and it was found that vortex shedding and flow separation around the wheelset were the key factors for the aerodynamic noise generation [10]. The flow-induced noise from the main sources of a full-scale simplified high-speed train was studied numerically using the latticeBoltzmann method (LBM) along with a turbulence wall-function approach to provide general information on the aerodynamic and aeroacoustic behaviour, although verification by experimental measurements was required to improve confidence [11]. In contrast, modelling numerically some simplified geometries can reveal more details of the flow behaviour and the corresponding aeroacoustic mechanisms for some main noise-generating components of high-speed trains. Moreover, these numerical simulations can be performed with affordable computer resources and verified by experimental measurements. Results from these model cases can be used to determine the relative importance of various aerodynamic noise sources and establish an efficient method to predict the aerodynamic noise from high-speed trains [12].

It is still very difficult to predict aerodynamic noise in an industrial context due to large computational resources required for unsteady numerical simulations [1]. Generally, the high-speed train bogies contain many components exposed to flow and the air flow 
passing through the bogie area is essential for the cooling of brakes, motors and wheels. Recently, numerical modelling of the aerodynamic noise generation from a simplified bogie was carried out and some preliminary results were investigated [13]. The simulation commenced with the flow behaviour and aeroacoustic characteristics around an isolated wheelset before progressing to tandem wheelsets and then a simplified bogie. By comparison, the current paper aims to study more details of the flow behaviour and the corresponding aeroacoustic mechanisms of the scaled simplified bogie. Moreover, the aerodynamic noise predictions are verified by experimental measurements from an anechoic open-jet wind tunnel.

\section{Numerical Method}

A two-stage strategy of computational fluid dynamics (CFD) and computational aeroacoustics (CAA) methods is employed in the simulations. Aerodynamically, highspeed trains are operating within the low Mach number flow regime, for example at 300 $\mathrm{km} / \mathrm{h}$ the Mach number is about 0.25 . The incoming flow speed simulated here is 30 $\mathrm{m} / \mathrm{s}$ (corresponding to a Mach number of 0.09) and thereby the compressibility effects may be neglected in studying the hydrodynamics of the flow field. Moreover, at low Mach numbers the dominant noise sources are the dipole sources from wall pressure fluctuations, which can be predicted essentially through incompressible flow modelling. Therefore, the unsteady, incompressible Navier-Stokes equations are used to solve the flow field. The continuity and momentum equations in tensor notation are

$$
\begin{gathered}
\frac{\partial u_{i}}{\partial x_{i}}=0 \\
\frac{\partial u_{i}}{\partial t}+u_{j} \frac{\partial u_{i}}{\partial x_{j}}=f_{i}-\frac{1}{\rho} \frac{\partial p}{\partial x_{i}}+v \frac{\partial^{2} u_{i}}{\partial x_{j} \partial x_{j}}
\end{gathered}
$$

where $x_{i}$ represents the Cartesian coordinates in three directions for $i=1,2,3$. $p$ is the pressure, $\rho$ is the density, $v$ the kinematic viscosity, $f_{i}$ is the body force and $u_{i}$ the flow velocity. Here $\rho$ and $v$ are constants for incompressible flow. The open source software OpenFOAM-2.2.1 is employed to solve the governing equations. A second-order accurate scheme is used for the spatial derivatives and the temporal discretization follows a second-order fully implicit scheme. The delayed detached-eddy simulation (DDES) model based on the one-equation Spalart-Allmaras (S-A) turbulence model is employed for all simulations. 
DDES is an extension of the detached-eddy simulation (DES) method which combines the large-eddy simulation (LES) in the main flow region with the Reynolds-averaged Navier-Stokes (RANS) approach in the boundary layer region close to the solid objects. Successful applications of DES to aeroacoustic problems have been confirmed by the BANC-I workshop results with different codes [1]. DDES has been developed to avoid grid-induced separation and preserve the RANS mode throughout the boundary layer [14]. In simulations of turbulent flow using DDES, the switch between RANS and LES is controlled by a redefined length scale which depends on both the geometrical parameters (such as the cell wall distance and grid spacing) and the time-dependent eddy-viscosity field. If a point is indicated inside a boundary layer, the RANS treatment is kept active; and when the massive flow separation occurs, the LES mode is applied.

The near-field unsteady flow computation provides acoustic sources which are fed to an acoustic analogy for far-field noise prediction. Assuming the fluid parameters being generalized functions and utilizing conservation laws with generalized derivatives, the formal solution of the Ffowcs Williams-Hawkings (FW-H) equation [15] may be written as

$$
\begin{aligned}
p^{\prime}(\mathbf{x}, t)=\frac{\partial}{\partial t} \int_{f=0} & {\left[\frac{Q_{i} n_{i}}{4 \pi|\mathbf{x}-\mathbf{y}|}\right]_{\tau_{e}} d S-\frac{\partial}{\partial x_{i}} \int_{f=0}\left[\frac{L_{i j} n_{j}}{4 \pi|\mathbf{x}-\mathbf{y}|}\right]_{\tau_{e}} d S } \\
& +\frac{\partial^{2}}{\partial x_{i} x_{j}} \int_{f>0}\left[\frac{T_{i j}}{4 \pi|\mathbf{x}-\mathbf{y}|}\right]_{\tau_{e}} d V,
\end{aligned}
$$

where []$_{\tau_{e}}$ denotes the evaluation at the emission time $\tau_{e}$. The control surface ' $S$ ' is typically described by $f(\mathbf{x}, t)=0$ such that $\widehat{\boldsymbol{n}}=\nabla f$ is the unit normal vector pointing outward to the surface. The acoustic pressure $p^{\prime}(\mathbf{x}, t)$ represents the pressure fluctuation perceived by an observer located at position $\mathbf{x}$ and at observer time $t$. The equivalent source terms under the integral sign are: $Q_{i}$ and $L_{i j}$ thickness and loading noise; $T_{i j}$ the Lighthill stress tensor [16]. Owing to a low Mach number flow around the geometries here, sound radiation from the quadrupole source (the last term in Equation (3)) is neglected and Farassat's Formulation $1 \mathrm{~A}$ with an integral solver based on the retarded time approach is employed to solve the FW-H equation [17].

\section{Simulation Setup}


In this study, a simplified bogie is considered at scale 1:10, which corresponds to the model used in wind-tunnel measurements for comparison with the numerical calculations. The flow speed of $30 \mathrm{~m} / \mathrm{s}$ is also chosen to match that applied in the experiments. The wheels are approximated as flat-sided discs, which may be seen as a simplification to wheels fitted with the wheel-mounted brake discs used on the power bogie of high-speed trains. Fig. 1 shows the simplified bogie model at scale 1:10. The axle has a diameter $(d)$ of $17.5 \mathrm{~mm}$ and the wheel diameter $(D)$ is $92 \mathrm{~mm}$. The wheelbase (centre-to-centre length of two axles) is $252 \mathrm{~mm}$ which is about 14 times the axle diameter. A tandem-wheelset case with the bogie frame removed is also considered, which has the same configuration as the bogie apart from the frame.

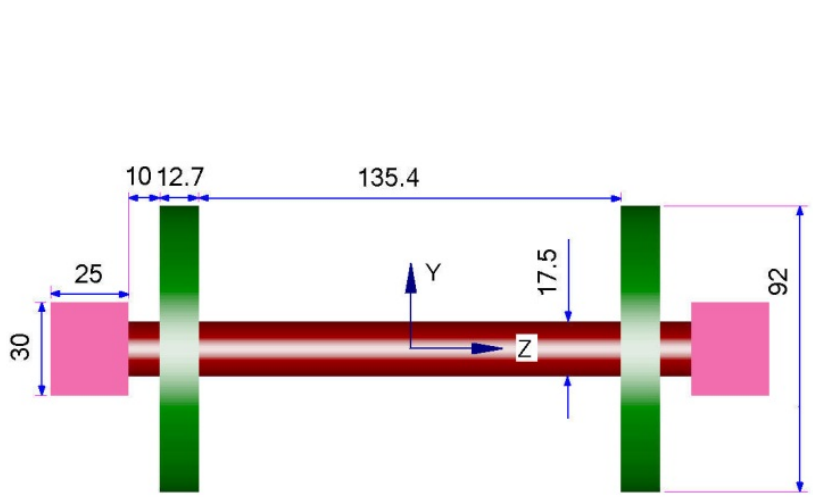

(a) Front view

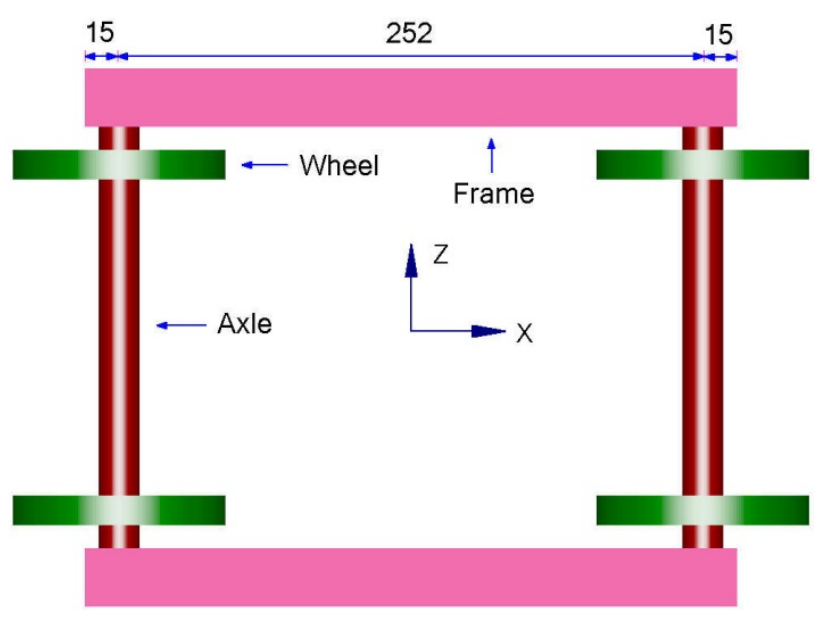

(b) Top view

Fig. 1. Simplified bogie model (1:10 scale, dimensions in millimetres)

The bogie geometry is symmetrical in the axle mid-span ( $x-y$ plane) where the influence from the wheel and frame is small; therefore it is reasonable to consider only half of the bogie and make use of the symmetry of the geometry to reduce the computation cost. The computational domain for the bogie case has dimensions of $17.7 D, 10 D$ and $6.3 D$ ( $D$ is the wheel diameter) along the streamwise $(x)$, vertical $(y)$ and spanwise $(z)$ direction, respectively, yielding a blockage ratio (defined as the ratio of the projected bogie area to the domain cross-section area) of $0.6 \%$, which is well within the prescribed range for cylinder flow (less than 3\%) [18]; and the outlet boundary is far enough downstream to have negligible influence on the near-wake flow around the bogie. 
Based on the results of a grid convergence study given in the Appendix A for flow around a circular cylinder, a fully structured mesh is generated around the bogie (displayed in Fig. 2) with resolutions similar to the cylinder 'Baseline' grids. The cell size on the axle surface is implemented as $0.42 \mathrm{~mm}$ around the perimeter and $0.88 \mathrm{~mm}$ in the spanwise direction. The maximum cell size on the wheel surface is $0.98 \mathrm{~mm}$. The mesh in the corner area between the wheel and axle is refined with double grid points in the wheel radial direction and the axial direction of the axle. The cell size of the frame is around $0.9 \mathrm{~mm}$. The distance from the solid surfaces to the first grid point is set as $1 \times 10^{-5} \mathrm{~m}$ and stretched with a growth ratio of 1.1 in the wall-normal direction inside the boundary layer. This yields a maximum value of $y^{+}$(the dimensionless first-cell spacing, $y^{+}=\frac{y u_{\tau}}{v}$ where $y$ is the distance from the wall, $u_{\tau}$ the friction velocity and $v$ kinetic viscosity) less than 1 for all cases which ensures that the boundary layer is resolved properly and the turbulence model employed can account for the low-Reynolds number effects inside the viscous sublayer. This grid generation strategy results in a fully blockstructured mesh in the entire domain with a total number of grid points of 14.6 million. Simulations are run with a physical timestep size of $5 \times 10^{-6} \mathrm{~s}$ followed by $1 \times 10^{-5} \mathrm{~s}$ which gives an adequate temporal resolution for the implicit time marching scheme used with a Courant-Friedrichs-Lewy (CFL) number of less than 1 within most part of the computational domain and the maximum value of 2 within the whole computational domain. Similarly, following the same mesh generating approach, a fully blockstructured mesh with 11.7 million grid points in the entire domain is generated for the tandem-wheelset case.

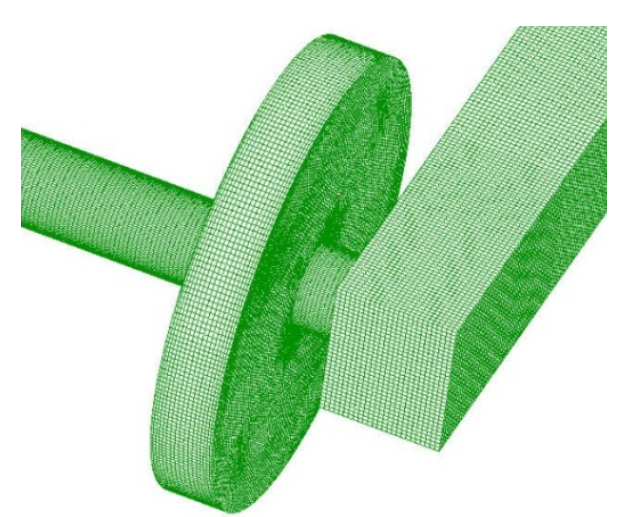

(a) Bogie surface

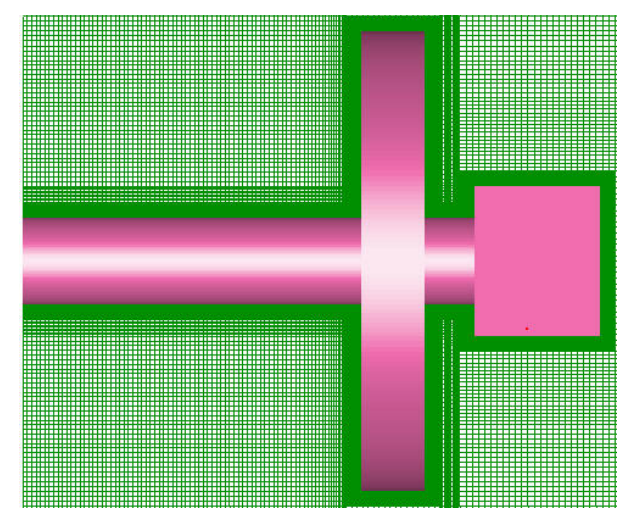

(b) Wheelset mid-plane

Fig. 2. Structured mesh topology around the bogie 
The boundary conditions applied are as follows: the upstream inlet flow is represented as a steady uniform flow $\left(U_{0}\right)$ with a low turbulence intensity corresponding to the experimental measurements from an anechoic open-jet wind tunnel; the top, bottom, axle mid-plane and side boundaries are specified as having symmetry boundary conditions which are equivalent to zero-shear slip walls; A pressure outlet with zero gauge pressure is imposed at the downstream exit boundary and all solid surfaces are defined as stationary no-slip walls. The Reynolds number (based on the freestream properties and the axle diameter) of the simplified bogie and tandem-wheelset cases is 36,000 .

\section{Aerodynamic Results}

In order to understand the flow behaviour around the bogie, the simulation results are presented for the instantaneous iso-surfaces of $Q$-criterion and vorticity fields; then, the gauge pressure at different positions in the wake area, the fluctuating lift and drag coefficients from the bogie and its components are compared and analyzed.

\subsection{Flow field}

Fig. 3 visualizes the iso-surfaces of the second invariant of the velocity gradient $Q$ to get an overview of the unsteady flow developed around the bogie. Defined as $Q=$ $\frac{1}{2}\left(\Omega_{i j} \Omega_{i j}-S_{i j} S_{i j}\right)$ where $S_{i j}=\left(u_{i, j}+u_{j, i}\right) / 2$ and $\Omega_{i j}=\left(u_{i, j}-u_{j, i}\right) / 2$ are the symmetric and antisymmetric components of velocity gradient respectively, the second invariant of the velocity gradient $Q$ identifies the vortical structures in turbulent flow. Here the isosurfaces are plotted at a normalized value of 25 (based on $Q /\left[\left(U_{0} / D\right)^{2}\right]$, where $D$ is the wheel diameter). They are coloured by the non-dimensional velocity magnitude. It shows that the flow around the bogie is complex. Flow separation occurs at the upstream wheelset and at the front edges of the frame. The following wake area is dominated by strong vortical structures of various scales at different levels of turbulence. Distinct features are observed in different regions of the flow field. Both streamwise and spanwise vortices are generated behind the front axle where the quasitwo-dimensional spanwise vortices start to shed and are subsequently developed into large scale organized streamwise vortices with high turbulence levels. These vortices are convected downstream and impinge on the downstream components, resulting in 
the wake region behind the downstream axle having less coherent large-scale structures and the corresponding vortex shedding being dominated by small-scale vortices with various orientations. Additionally, it can be seen that streamwise 'rib' vortices are developed behind the upstream axle inside the wheels and distributed obliquely along the streamwise direction since the turbulent flow develops more rapidly close to the mid-span axle region due to much less blockage far away from the wheelframe junction area within the bogie.

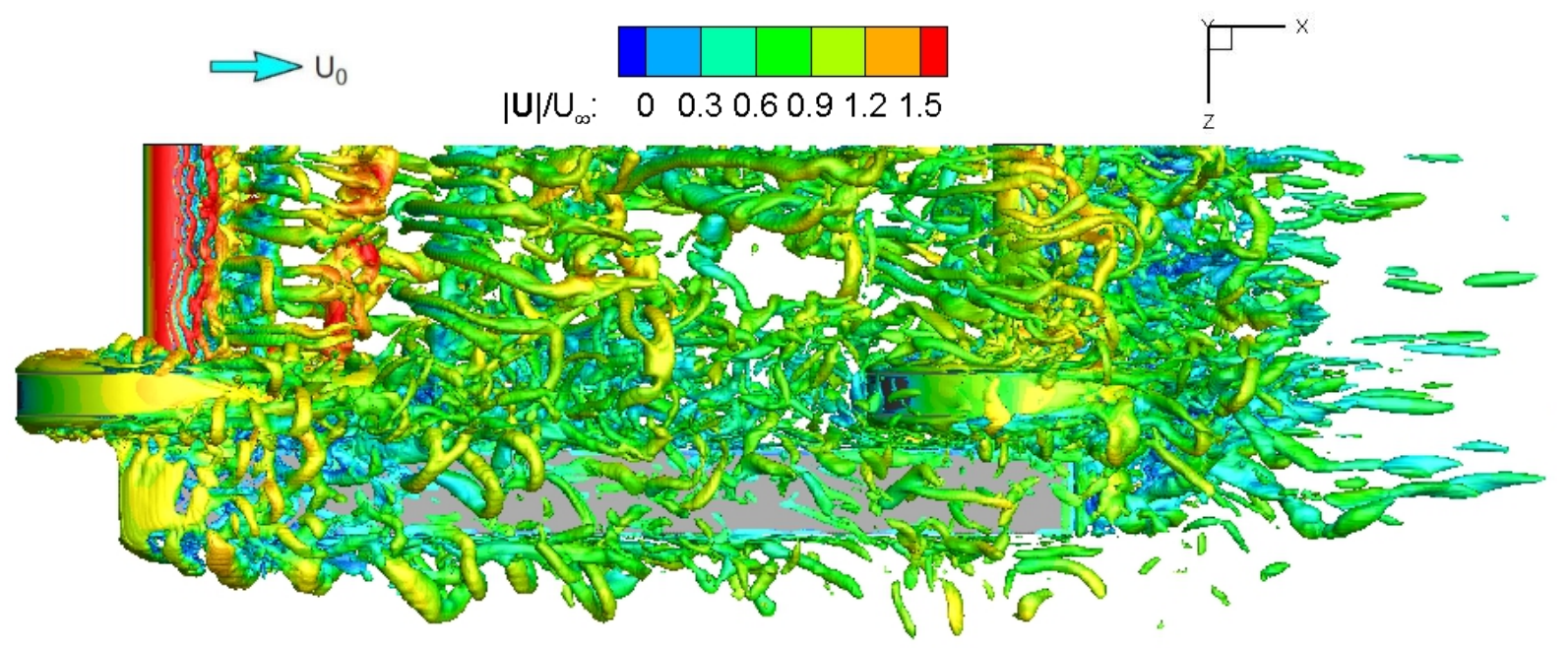

Fig. 3. Iso-surface of the instantaneous normalized $Q$-criterion (at value of 25)

Fig. 4 displays the instantaneous non-dimensional spanwise vorticity field $\left(\omega_{z}=\right.$ $(\partial V / \partial x-\partial U / \partial y) D / U_{\infty}$, where $D$ is the wheel diameter and $U_{\infty}$ the freestream velocity) for the simplified bogie case. Fig. 4(a) shows the spanwise vorticity field contours in a mid-plane between the wheel inner surface and axle mid-span. As mentioned earlier, the front and rear axles are separated by the centre-to-centre distance of 14 times the axle diameter. Since the downstream axle is sufficiently far from the upstream one, the rear axle is well outside the recirculation region of the front axle wake and vortex shedding may be generated from both axles, referred as co-shedding pattern for tandem-cylinder flow. It is shown that the downstream axle experiences a periodic impingement of vortices shed from the upstream axle; and consequently, the flow around the downstream axle becomes highly unsteady. The incident vortices are greatly deformed as they are swept over the downstream axle; thereby, all vortices are mixed up behind the rear axle, leading to the synchronized behaviour of the downstream axle wake. Additionally, the separation angle $\theta$ (defined in the clockwise direction with zero 
at the axle front stagnation point) around the rear axle is $88.2^{0}$ compared to $82.1^{0}$ for the front axle. Thus, the flow keeps attached to the rear axle longer than to the front axle as the turbulent boundary layer is developed around the downstream axle due to the interactions from upstream flow.

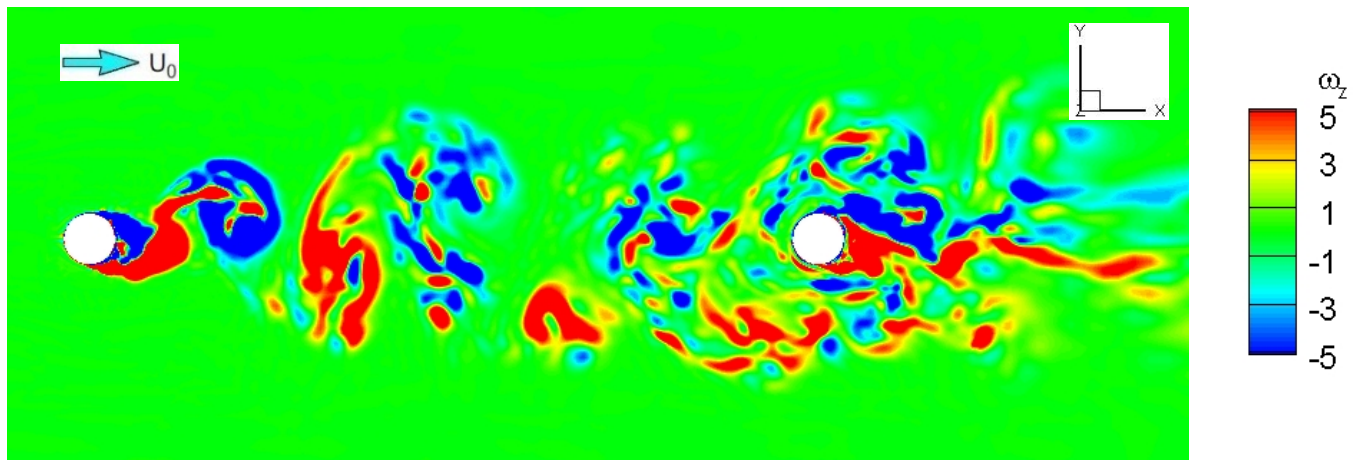

(a) Mid-plane between the wheel inner surface and axle mid-span

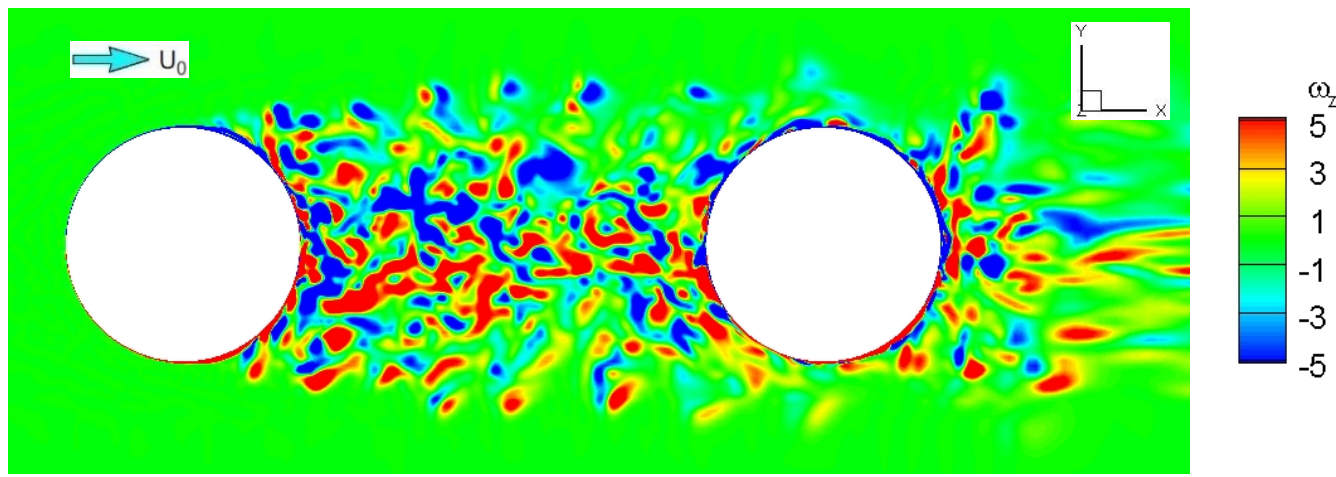

(b) Wheel mid-span

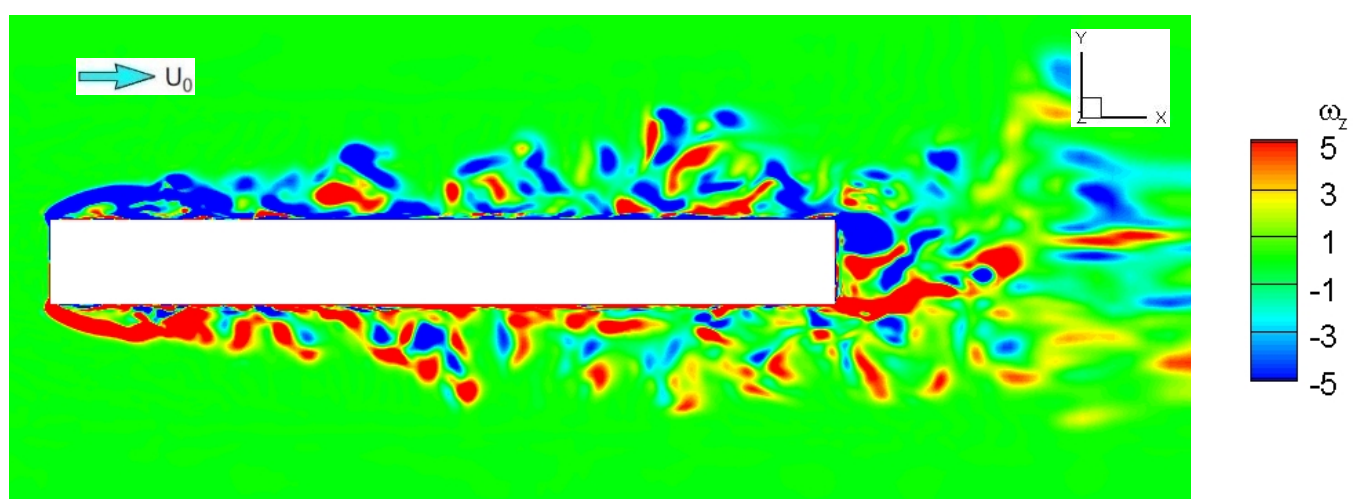

(c) Frame mid-span

Fig. 4. Contours of the instantaneous spanwise vorticity field in a vertical plane (side views) 
Compared to the wakes developed behind the axles, the flow around the wheels shows different characteristics, as displayed in Fig. 4(b) in terms of contours of the instantaneous spanwise vorticity field $\left(\omega_{z}\right)$ along the wheel mid-span. This shows that the wakes behind both the upstream and downstream wheels are highly unsteady with lower turbulence levels since no large-scale coherent vortex shedding occurs there. Compared to the axle, the wheel has a very small aspect ratio (the ratio of span to diameter) with sharp edges. Thus, the wake behind the upstream wheel is different from that of the upstream axle. The flow separates from the upstream wheel front edges and interferes with the flow separated on the wheel tread; therefore, the coherent vortex shedding, seen behind the front axle, cannot be formed behind the front wheel and the wake developed there becomes fully three-dimensional. The wheelbase is about three times of the wheel diameter, leading to the downstream wheel being strongly influenced by the wake of the upstream wheel. The incident vortices convected from the upstream geometries impinge on and interfere with the vortices separated from the downstream wheel, forming a highly unsteady wake with less organized flow structures around the downstream wheel.

The contours of the instantaneous spanwise vorticity field $\left(\omega_{z}\right)$ along the frame midspan are displayed in Fig. 4(c). This shows that flow separation occurs at the front edge of the frame, interfering with the boundary layer developed on the frame top and bottom surfaces. The vortices generated and convected downstream along the frame are separated again at the frame trailing edges, resulting in a wake area with unsteady shear flow. 


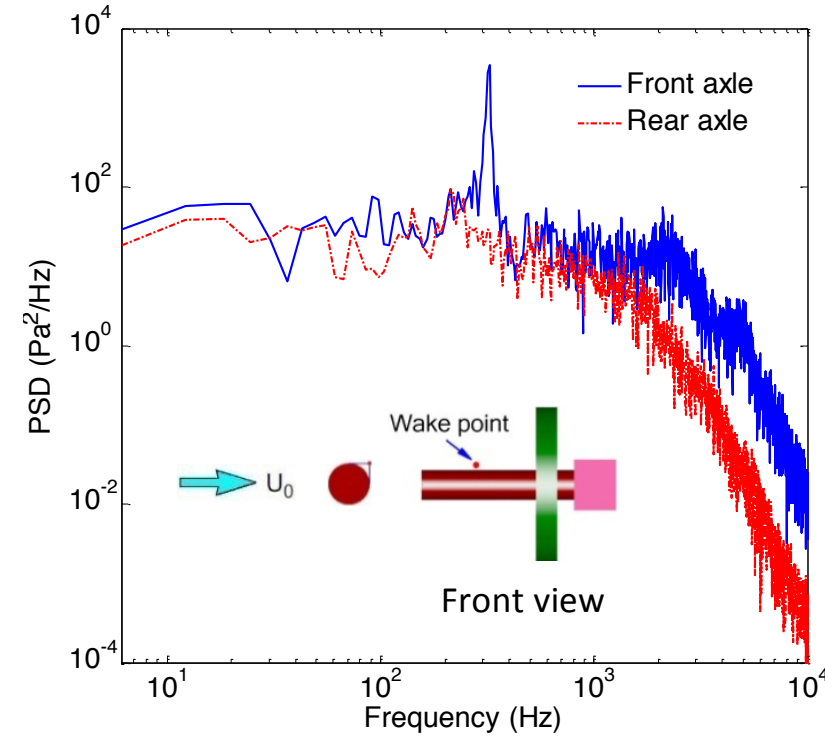

(a) Axle mid-span

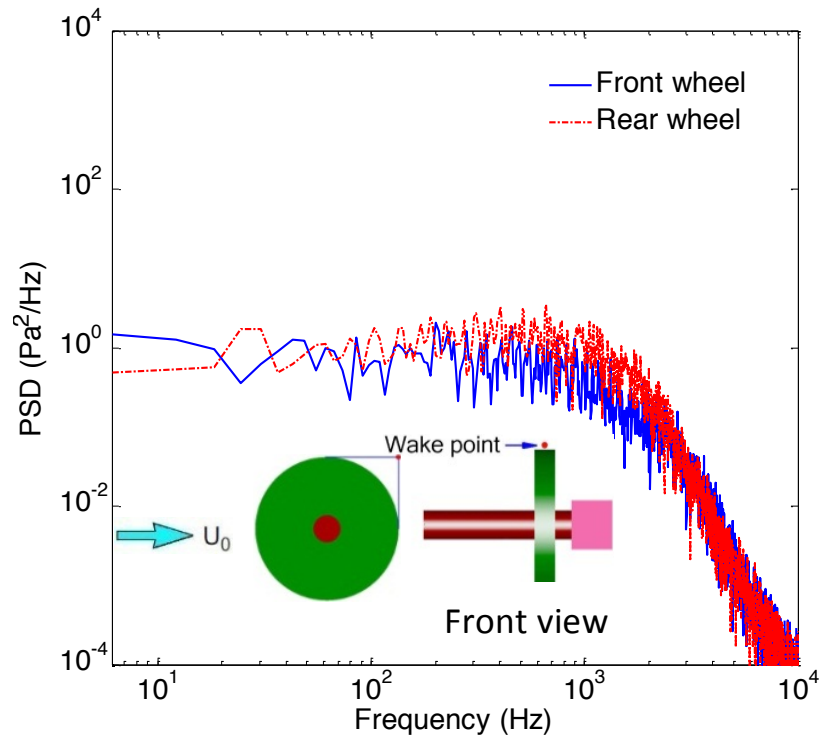

(b) Wheel mid-span

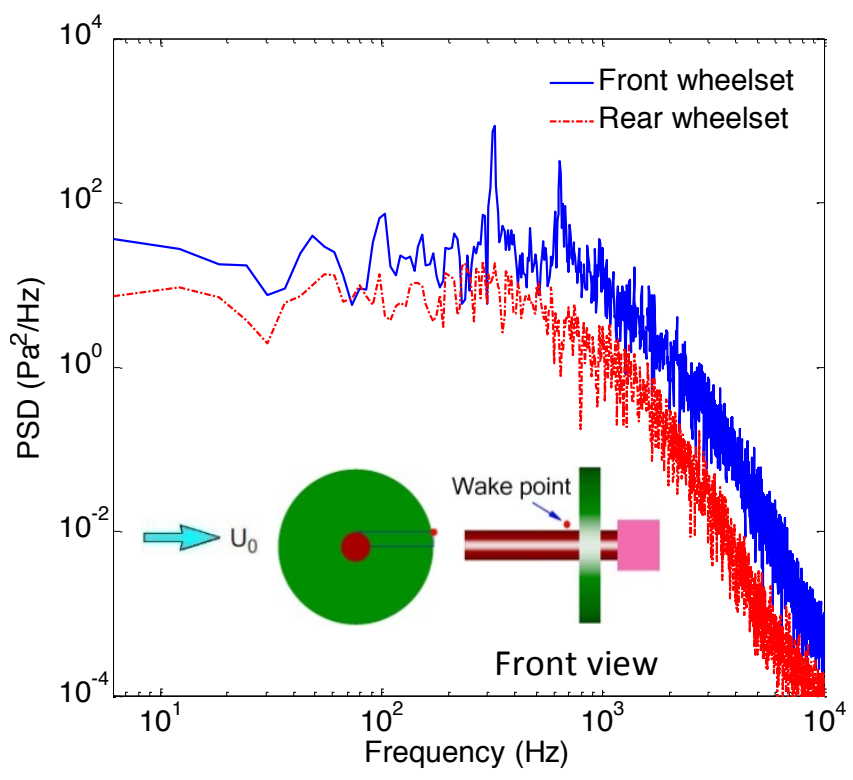

(c) Inner wheel-axle corner

Fig. 5. Power spectral densities of pressure at bogie wake positions 
The distinct characteristics of the wakes behind the axle and wheel can also be revealed by the flow unsteadiness. Fig. 5 shows power spectral densities (PSDs) of the gauge pressure at different positions in the wakes of the front and rear wheelsets: behind the axle, the wheel and at the inner wheel-axle corner. For the point one axle radius above and behind the front axle in the mid-plane between the wheel inner surface and axle mid-span, a tonal peak appears in the spectrum at $324 \mathrm{~Hz}$, as seen in Fig. 5(a). This frequency is associated with the vortex shedding from the front axle, and the corresponding Strouhal number (non-dimensionalized by the freestream velocity and the axle diameter) is 0.19 , typical of vortex shedding from a cylinder in subcritical regime. By contrast, the pressure signal at a similar position behind the rear axle is more broadband with no tonal peak and at lower amplitudes. Fig. 5(b) illustrates the spectra of the pressure at the points one wheel radius away from the top of the front and rear wheels at wheel mid-span. The PSDs are broadband with no evident peak and their amplitudes are close to each other, indicating that the flow behind the front and rear wheels is fully turbulent and the wake contains similar turbulence structures with various scales. Fig. 5(c) shows the results of points located at $13 \mathrm{~mm}(0.75 d)$ away from the wheel inner rim in line with the top of the axles. From the front wheelset inner wheel-axle corner, two peaks appear at the frequencies of $324 \mathrm{~Hz}$ and $641 \mathrm{~Hz}$ : the tonal peak (first harmonic) is related to the axle vortex shedding as mentioned earlier; and the second harmonic with twice the shedding frequency corresponds to the interaction between the periodic vortex shedding from the axle and the wheel inner surface, resulting in the surface fluctuations developed on the wheel surface downstream of the axle. The flow interactions mainly change the fluctuating drag from the wheelset and introduce a tonal peak at double the shedding frequency. It is also noted that due to the influence of the turbulent wake interaction effects, the spectrum of the pressure signal at the point in the inner wheel-axle corner of the rear wheelset is more broadband and with much lower amplitude than that from the front wheelset.

\subsection{Lift and drag coefficients}

All simulations were run for $0.1 \mathrm{~s}$ to allow the flow to develop fully before collecting the time series of flow quantities. The lift and drag coefficients corresponding to the forces normal to and along the flow direction are non-dimensionalised by $\left(\frac{1}{2} \rho_{0} U_{\infty}^{2} A\right)$, where $A$ is the projected frontal cross-section area of the bogie. The data are divided into three 
$50 \%$ overlapping segments. Mean and root-mean-square fluctuating lift and drag coefficients are calculated for each segment, which can be used to check the flow development and the convergence of the statistics collected. Table 1 summarizes the RMS and mean results from the three overlapping time windows. It is shown that the variations of the RMS values between each segment are less than $3 \%$ and the discrepancies in the mean drag coefficient are less than $0.1 \%$. The mean lift coefficient is always less than 0.005 for each segment. Therefore, it is suggested that the transient flow field has become statistically steady. Note that the RMS lift coefficient is about six times larger than the RMS drag coefficient, suggesting the oscillation of unsteady force is much higher normal to the flow direction.

\begin{tabular}{l|ccccc}
\hline \multicolumn{2}{l}{} & $\begin{array}{c}\text { Segment1 } \\
(0.1-0.35 s)\end{array}$ & $\begin{array}{c}\text { Segment2 } \\
(0.225-0.475 s)\end{array}$ & $\begin{array}{c}\text { Segment3 } \\
(0.35-0.6 s)\end{array}$ & $\begin{array}{c}\text { Total length } \\
(0.1-0.6 s)\end{array}$ \\
\hline $\begin{array}{l}\text { RMS } \\
\text { value }\end{array}$ & Fluctuating lift & 0.2289 & 0.2280 & 0.2243 & 0.2267 \\
\hline $\begin{array}{l}\text { Mean } \\
\text { value }\end{array}$ & Drag coefficient & 1.4991 & 1.4978 & 1.4986 & 1.4989 \\
\hline
\end{tabular}

Table 1. Root-mean-square and mean values of lift and drag coefficients

The PSDs of the fluctuating lift coefficients of the symmetrical half bogie simulated and its components (frame, front and rear wheelsets) are presented in Fig. 6. A tonal peak (Fig. 6a) appears in the lift coefficient of the bogie at $324 \mathrm{~Hz}$, giving a Strouhal number (non-dimensionalized by the freestream velocity and the axle diameter) of 0.19 . As discussed earlier, this peak is related to the vortex shedding around the axle of the upstream wheelset, which appears in the spectrum of the front wheelset shown in Fig. 6(b). A broad hump between $160 \mathrm{~Hz}$ and $390 \mathrm{~Hz}$ can be observed in the lift coefficient of the rear wheelset (Fig. 6b), which may correspond to the mixture of the incident vortices convected inside the bogie with the eddies generated by the flow passing the rear wheelset. Moreover, the contribution to the oscillating lift force from the frame is much smaller (Fig. 6a) compared with other components of the bogie. 


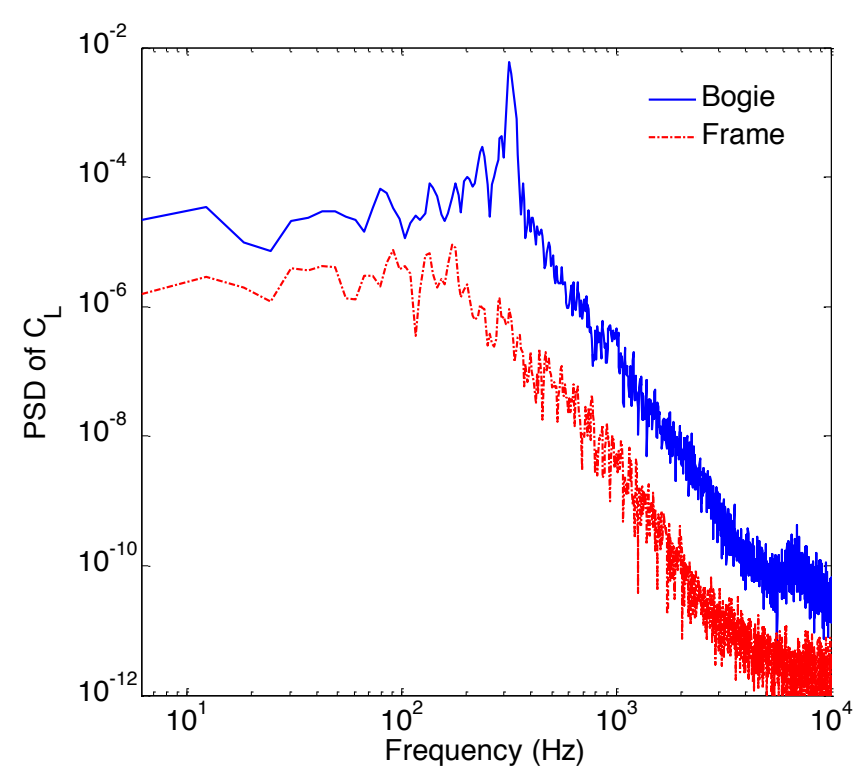

(a) Bogie and frame

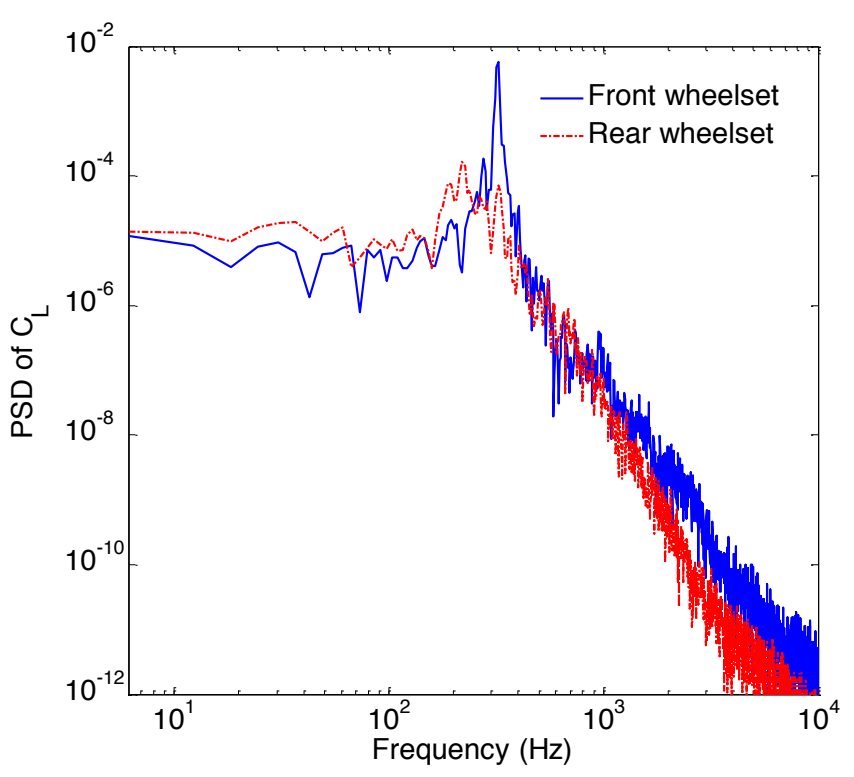

(b) Front and rear wheelsets

Fig. 6. Power spectral densities of lift coefficients

Fig. 7 shows the PSDs of the fluctuating drag coefficients of the symmetrical half bogie and its components. A peak appears in the drag coefficient of the bogie and the front wheelset at $641 \mathrm{~Hz}$, which is twice the frequency of the tonal peak in the lift coefficient while at a much lower amplitude. This peak corresponds to the fluctuating drag induced by the vortex shedding from the upstream axle. The alternate periodic vortex shedding from the front axle interacts with the boundary layer developed on the wheel side surface and the unsteady flow separated from it; thus, the resulting wake induces a regular fluctuating drag around the front wheelset. It is noted that the spectra of the drag coefficient from the frame and the rear wheelset are broadband as there is no massive coherent vortex shedding developed around them. Compared with the front wheelset, the spectrum level of the rear wheelset is lower in most of the frequency range and the contribution to the fluctuating drag force from the bogie frame is small, about one order of magnitude smaller. 


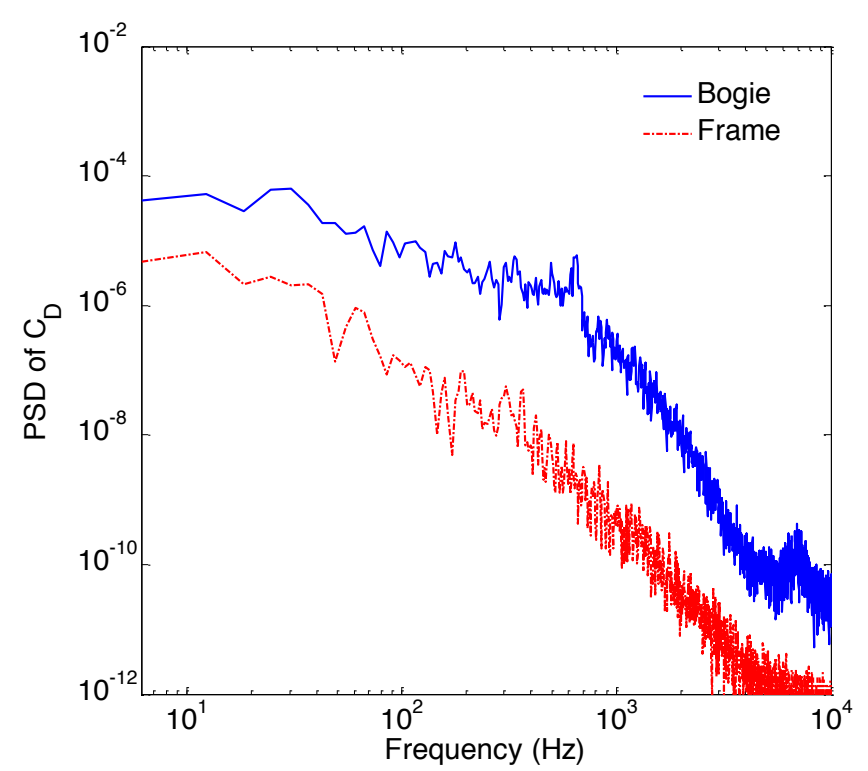

(a) Bogie and frame

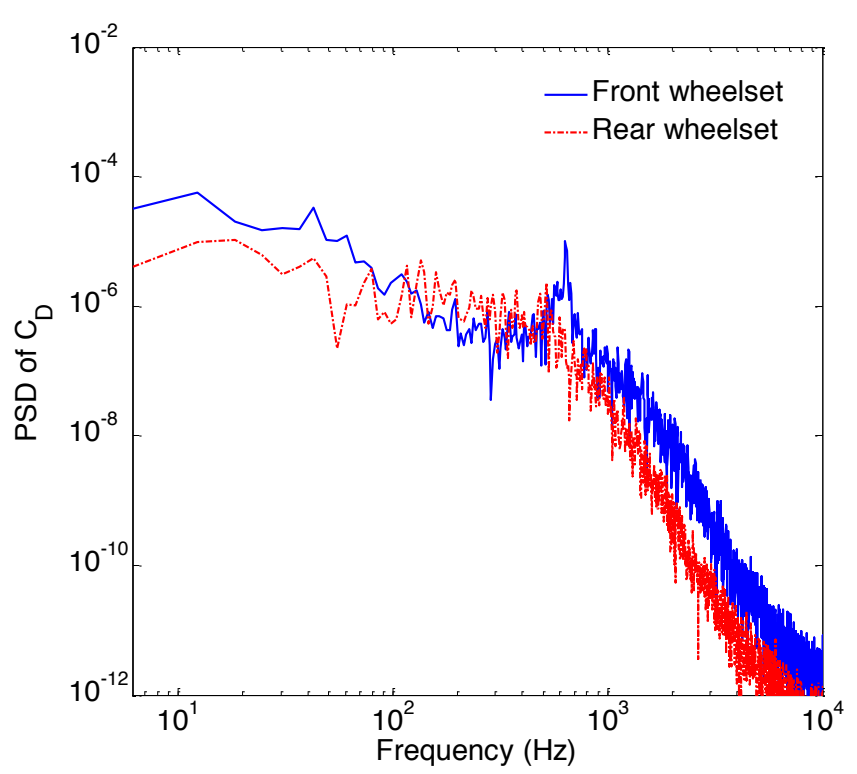

(b) Front and rear wheelsets

Fig. 7. Power spectral densities of drag coefficients

\subsection{Wall pressure fluctuations}

Fig. 8 displays the wall fluctuating pressure level in decibels $\left(L_{p}=10 \log \left(\overline{p^{\prime 2}} / p_{r e f}^{2}\right)\right.$, where $\overline{p^{\prime 2}}$ is mean-square fluctuating pressure and $p_{\text {ref }}$ is reference acoustic pressure $20 \mu \mathrm{Pa}$ ) on the bogie surface, which can be used to identify the potentially significant noise source regions. This shows that the surface pressure fluctuations are generally high in the rear half of the upstream axle due to vortex shedding and flow recirculation behind the front axle. As shown in Fig. 8(a), high pressure fluctuations appear on the upstream wheel inner surface behind the axle as a consequence of the flow interacting between the axle wake and the wheel side surface. Moreover, a crescent-shaped large pressure fluctuation region develops at the upstream half of the front wheel side surfaces owing to the flow separation generated from the wheel front sharp edges. All these high pressure fluctuations are the main contributors to the lift and drag dipoles from the front wheelset, as will be discussed in the next section. This also indicates that the massive vortex shedding generated from the front axle may potentially be a major contributor to the noise radiated from the bogie. Furthermore, the high pressure fluctuations can be seen around the downstream wheelset due to the flow impingement by the incoming vortex convected from the upstream geometry as well as the flow separation developed from the rear wheel front edges and the vortex shedding formed behind the rear axle. However, the distributions of the fluctuating pressure on the downstream wheelset are rather irregular and mainly concentrated in the upstream area. 
Compared to the wheelsets, the pressure fluctuations on the frame surface are much lower with small patches of high values distributed mostly in the upstream half. Additionally, the wall pressure fluctuations on the outer surfaces (Fig. 8b) of the wheel and frame have similar distribution properties to those on the inner surfaces of them as described above.

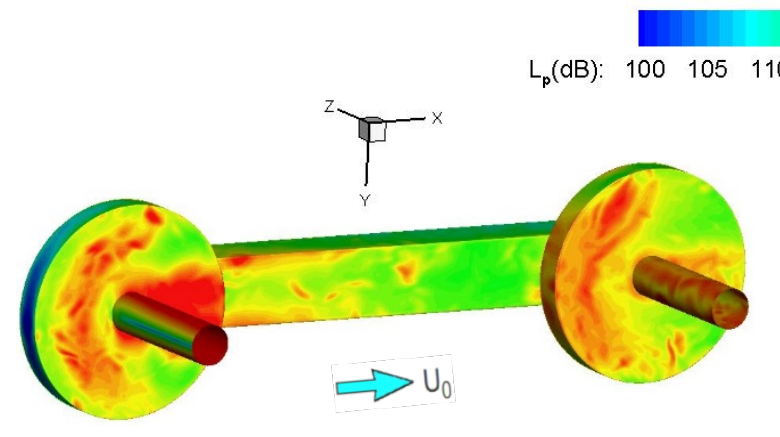

(a) Bogie inner surfaces

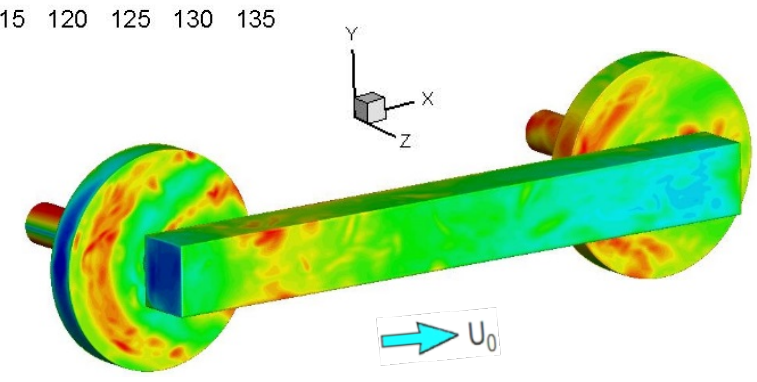

(b) Bogie outer surfaces

Fig. 8. Wall pressure fluctuation level of the simplified bogie

\section{Aeroacoustic Results}

The source data are collected for far-field noise prediction only when the transient flow field has become statistically steady. Based on the near-field unsteady flow data obtained from the CFD calculations, the far-field noise signals can be predicted by the FW-H acoustic analogy using equivalent acoustic sources. There are 104,928 panels (surface elements) around the bogie which account for the acoustic sources on the solid surfaces and 74,496 noise source panels for the tandem-wheelset case. The receivers are distributed uniformly along a circumference with radius $2.5 \mathrm{~m}$ at an interval of $5^{\circ}$ as sketched in Fig. 9 to measure the two-dimensional noise directivity through the upstream and downstream wheelset centreline respectively along the vertical $z-y$ plane. Additionally, equivalent circular-shaped receiver positions are defined in the horizontal $x-z$ plane (the coordinates referred to Fig. 1). This distance corresponds at full scale to $25 \mathrm{~m}$ as recommended for field measurements of railway noise. For the three-dimensional directivity calculation, the far-field observers are distributed on a spherical surface also with a radius of $2.5 \mathrm{~m}$, composed of 1,946 receivers with a resolution of $5^{\circ}$ for the azimuthal and polar angles. Therefore, the directivity characteristics of the source are obtained to represent the overall acoustic 
field through calculating the overall sound pressure level (OASPL) from the time history of the acoustic pressure at each specified receiver over the resolved frequency range.

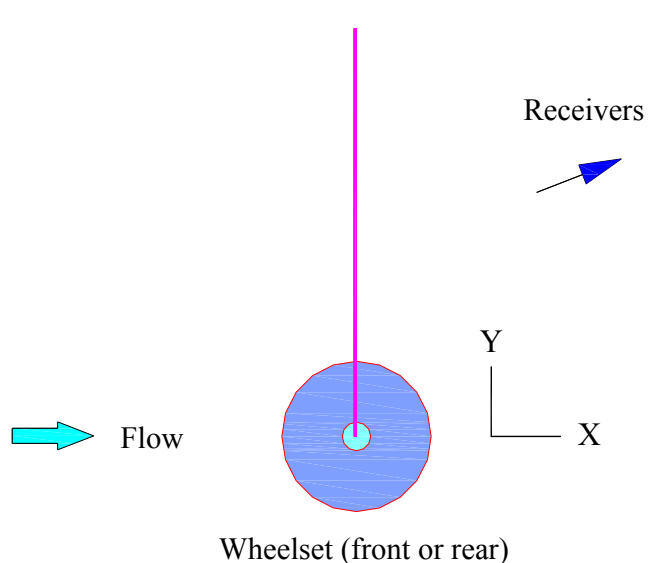

Fig. 9. Sketch of receiver locations

\subsection{Acoustic spectra computation}

Flow statistics on lift and drag coefficients in Section 4.2 suggest that the flow transient is washed out after $0.1 \mathrm{~s}$. The CFD simulations were run for $1.2 \mathrm{~s}$ corresponding to 21 times the flow-through time ( $L_{x} / U_{\infty}$, where $L_{x}$ is the computational domain length). The length of the time signal used as input to the FW-H method for noise calculation is related to the last $0.56 \mathrm{~s}$ of the computation. The PSD is computed from the predicted far-field noise time history by the Welch's method and averaged over $50 \%$ overlapping segments using a Hanning window applied to 5 segments [19], giving a frequency resolution of $6 \mathrm{~Hz}$. 


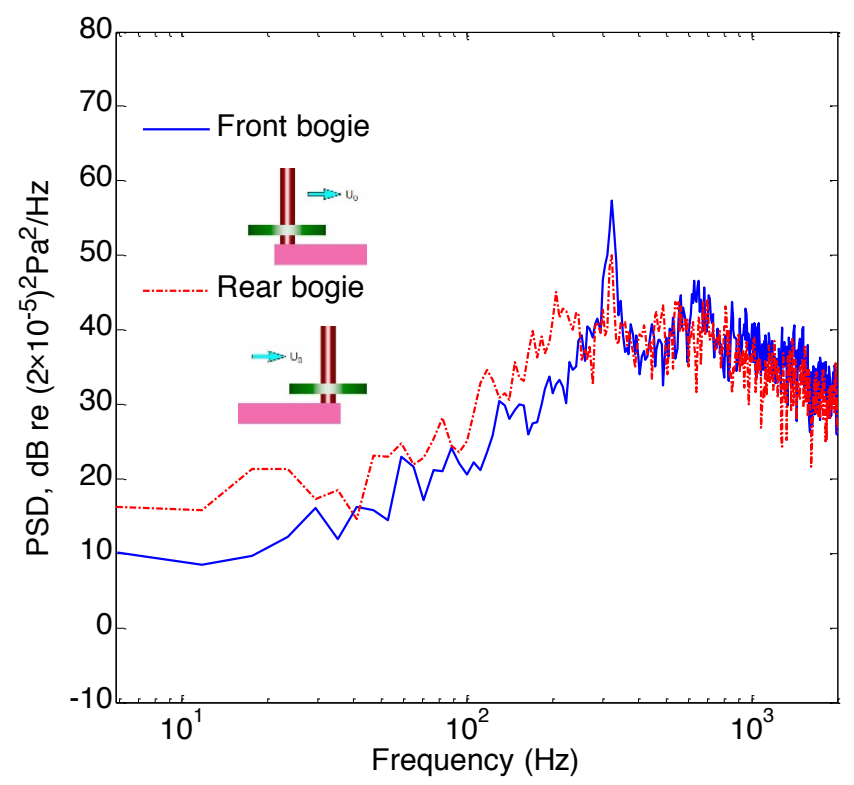

(a) Receiver 3

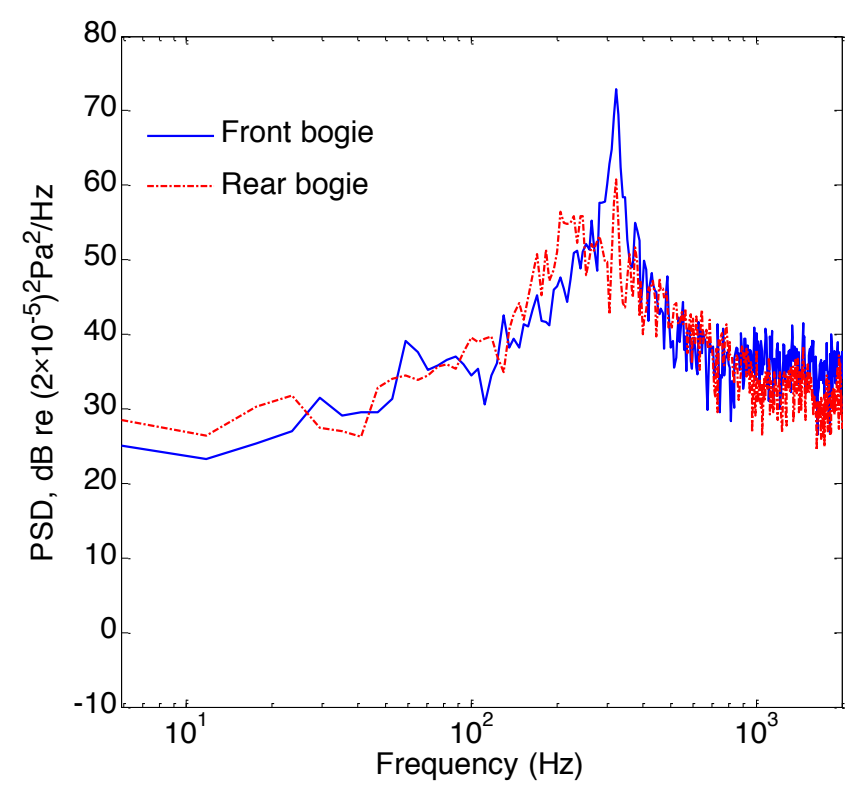

(b) Receiver 19

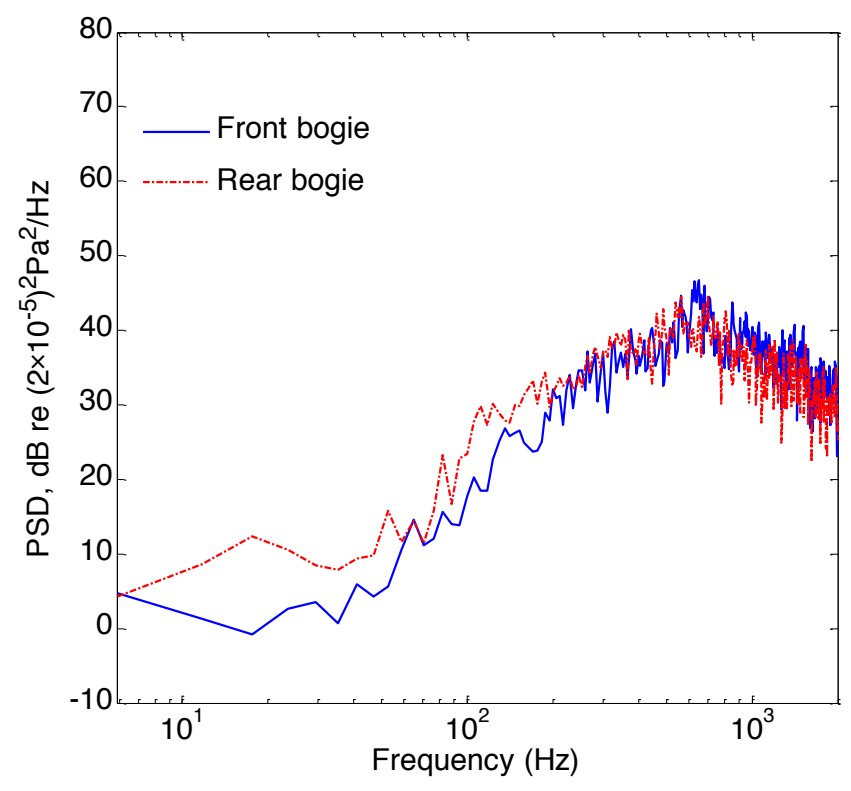

(c) Receiver 1

Fig. 10. Spectra of acoustic pressure on far-field receivers $\left(U_{\infty}=30 \mathrm{~m} / \mathrm{s}\right)$

Fig. 10 shows the spectra of the noise radiated from the front and rear bogies (i.e. the front and rear half parts of the half bogie symmetrical along the axle mid-span) at three receivers in the $z-y$ plane as described in Fig. 9. The spectra at receiver 3 , which is located $0.434 \mathrm{~m}$ above the axle axis, are plotted in Fig. 10(a). It can be seen that two tonal peaks appear at the frequencies of $324 \mathrm{~Hz}$ and $652 \mathrm{~Hz}$ since both lift and drag dipole components influence the radiated sound at this receiver, especially from the front bogie. The primary peak corresponds to the dominant peak in the oscillating lift 
forces related to the vortex shedding from the axle. The peak at the second harmonic is associated with the dominant peak in the oscillating drag force. As stated previously, the frequency of the fluctuating drag is twice that of the fluctuating lift. Additionally, the highest peak corresponding to the oscillation lift dipole component appears at receiver 19 as it is located right above the bogie, as shown in Fig. 10(b), while the largest drag dipole component occurs at receiver 1 (Fig. 10c) which is in the lateral direction. Compared with the front part of the bogie, the noise radiated from the rear part of the bogie is more broadband, resulting from the irregular and unsteady flow passing over it. In a turbulent inflow condition, the tonal peak related to the periodic vortex shedding generated around the downstream axle still can be observed on receivers 3 and 19; however, its amplitude is much lower compared to that of the upstream axle since the turbulent flow convected from the front axle becomes dissipated as indicated from Fig. 3 and the incoming flow speed around the rear axle is decreased to about $18 \mathrm{~m} / \mathrm{s}$ of the mean velocity compared to the inflow velocity of $30 \mathrm{~m} / \mathrm{s}$.

\subsection{Experimental verification}

Experimental measurements of sound generated by flow past the simplified bogie and tandem wheelsets were carried out in an open-jet anechoic wind tunnel in the University of Southampton. Measurements were taken for the same geometries as used in the numerical simulations. A model comprised of two half wheelsets connected by a bogie frame was attached to a baffle plate. The bogie was immersed within the core flow and the remaining parts in connection with the baffle plate were wrapped with foam to suppress the aerodynamic noise generated due to flow interaction with these regions. The experimental setup for the bogie case is displayed in Fig. 11(a) and for the tandemwheelset case in Fig. 11(b), where the test model is mounted in the working section on the rigid baffle. The same instruments of signal analyser and microphone array were used for both cases. The nozzle exit had a rectangular cross-section $(350 \mathrm{~mm} \times 500 \mathrm{~mm}$, width by height) and the flow speed was $30 \mathrm{~m} / \mathrm{s}$ with the turbulence level in the jet core below $0.3 \%$. The background noise from the anechoic chamber was measured and a noise of high spectra level was generated for frequencies below $100 \mathrm{~Hz}$, which was caused by the fans, the duct and the nozzle flow in the anechoic wind-tunnel. These frequencies were therefore not considered. In accordance with the numerical 
predictions, the receiver identified as 'top microphone' in Fig. 11(a) was located at (-18, $1375,31.3$ ) of which the dimensions were in millimetres and the coordinates (shown in Fig. 1) were defined with the origin at the centre of upstream axle outer end surface. The measurement was made with a sampling rate of $48 \mathrm{kHz}$ and contained a time signal of length $10 \mathrm{~s}$. Corresponding to the frequency resolution used in the simulation, the PSD of the experimental data was also computed by Welch's method with $6 \mathrm{~Hz}$ bandwidth.

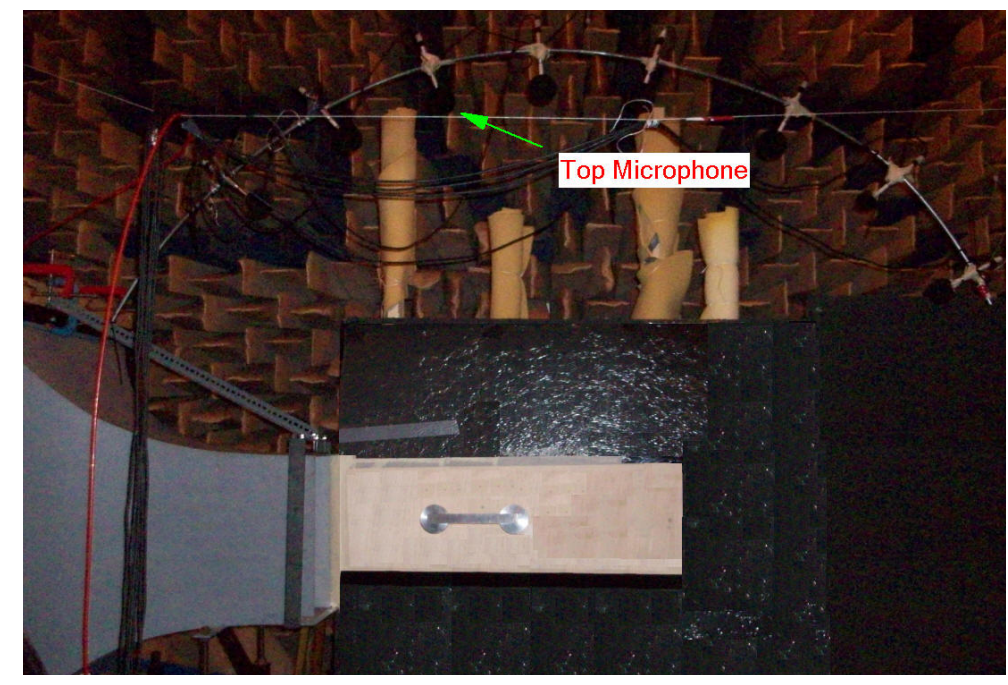

(a) Simplified bogie

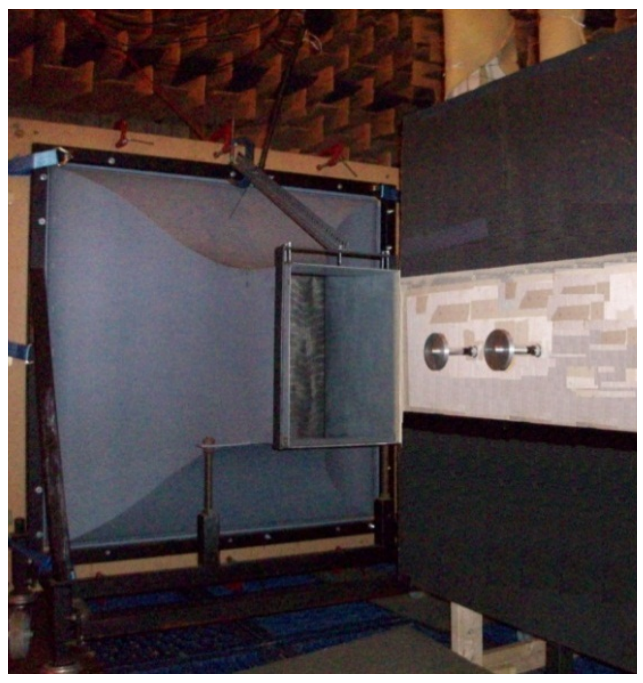

(b) Tandem wheelsets

Fig. 11. Experimental setup in the anechoic chamber

Fig. 12 displays the spectra of the radiated noise at the top microphone receiver (shown in Fig. 11) for the bogie and tandem-wheelset cases. The background noise of the anechoic chamber is also depicted and its noise level is by several orders of magnitude lower than those generated from the test geometries. Fig. 12(a) shows that the numerical prediction of the PSD of the far-field noise radiated from the bogie is in good agreement with the experimental measurements. The dominant frequency of the tonal noise from the experiment $(314 \mathrm{~Hz})$ is slightly lower (around $3 \%$ ) than the prediction $(324 \mathrm{~Hz})$. This is likely due to that the bogie has a relatively long configuration longitudinally and may not be situated completely inside the core region of the open-jet flow.

Fig. 12(b) compares the PSD level of the tandem-wheelset case between the noise prediction and experimental measurement. Very good agreement is achieved for the 
dominant frequency of the tonal noise and the shape of spectra, even a small peak around $940 \mathrm{~Hz}$ (third harmonic) is properly captured in the computation. It is noted that a broadened hump appears around $280 \mathrm{~Hz}$ for the bogie case and $250 \mathrm{~Hz}$ for the tandem-wheelset case in the measurements; however, no such hump is observed in the simulations of both cases. These broad peaks may correspond to the noise contribution from the interaction between the (front or rear) axle wake and the baffle plate, i.e. the vortex shedding in the axle wake region close to the baffle is decreased by the unsteady flow developed on the rigid plate and thereby the corresponding shedding frequency is reduced. The same phenomenon has been found in the experiments of flow-induced noise from wall-mounted cylinders [20]. Note that compared to the spectrum from the bogie, the low frequency broadened hump is larger from that of the tandem wheelsets, which is due to the stronger interaction between the vortex shedding and the baffle plate boundary layer produced around the axle-plate junction areas of the tandem wheelsets. As this broad peak amplitude is about $8 \mathrm{~dB}$ lower than the tonal peak level, its contributions to the overall sound pressure level are not significant. Moreover, compared with the experimental data, the tonal peak has a higher amplitude from the calculations in both cases. This is likely to be influenced by the rigid baffle used in the experiment which weakens the coherent vortex shedding from the axle while the symmetric boundary conditions with stronger spanwise uniformity are applied in the simulations. The OASPL in the frequency range between $100 \mathrm{~Hz}$ and $2 \mathrm{kHz}$ is calculated for the experimental measurements and it is slightly higher $(0.7 \mathrm{~dB})$ in the bogie case than the tandem-wheelset case, indicating that the noise contribution from the frame of the bogie is small. 


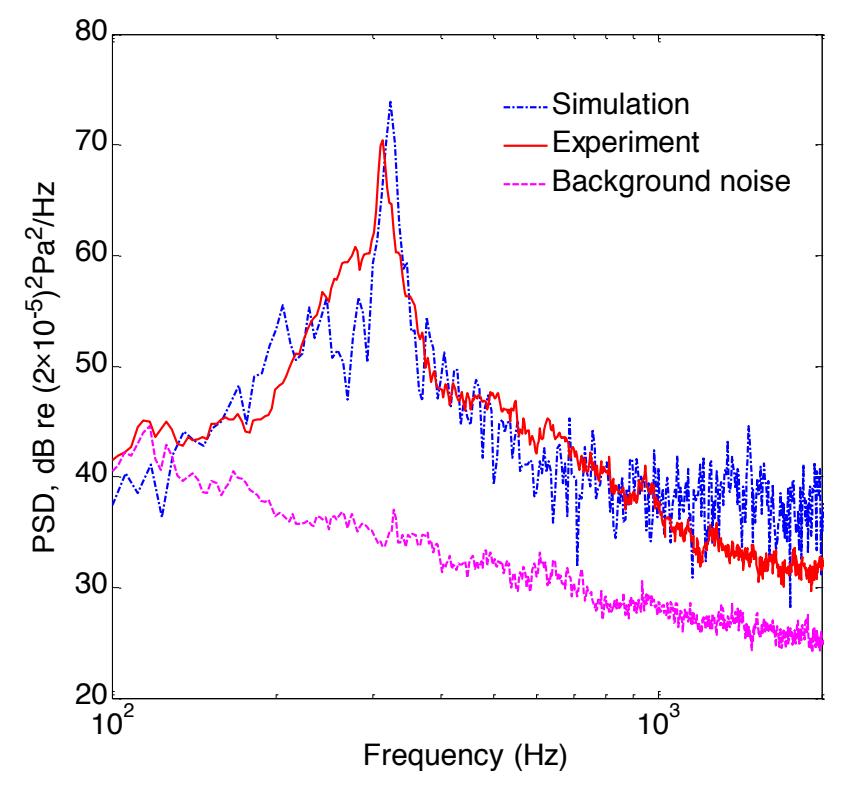

(a) Simplified bogie

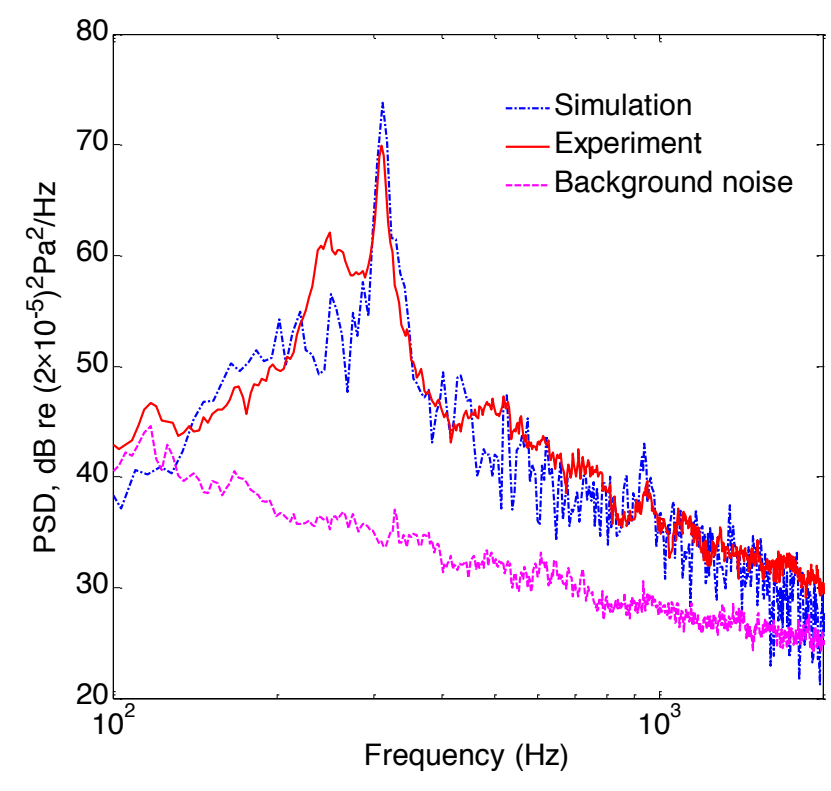

(b) Tandem wheelsets

Fig. 12. Comparisons of far-field noise spectra between simulation and experiment

\subsection{Acoustic directivity}

The directivity of the noise radiated to far-field is calculated based on the OASPL determined from the PSD in the frequency range below $2 \mathrm{kHz}$. Some numerical artefacts of the far-field noise simulation appear above $2.5 \mathrm{kHz}$ due to grid resolutions and are not included in calculating the OASPL as this is in excess of the main energycontaining frequency range. This can be confirmed from the experimental results (shown in Fig. 12) in which the noise level is much lower and drops remarkably for frequencies above $2 \mathrm{kHz}$. Based on the flow data from the half bogie as the sound source, the sound pressure levels from the whole geometry are given by $L_{p}=10 \log \left(10^{L_{p_{1}} / 10}+10^{L_{p_{2}} / 10}\right)$, where $L_{p_{1}}$ and $L_{p_{2}}$ are the sound pressure levels of two receivers located symmetrically along the symmetry plane and are assumed uncorrelated. In order to understand the noise contributions from various parts, the noise directivity patterns from different geometries and corresponding components are displayed and compared below. 


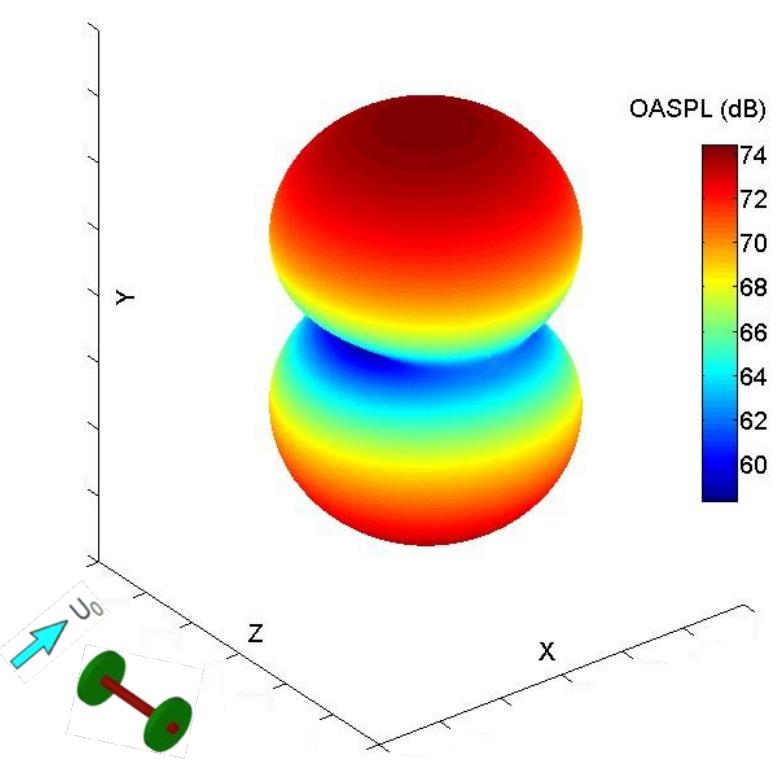

(a) Front wheelset

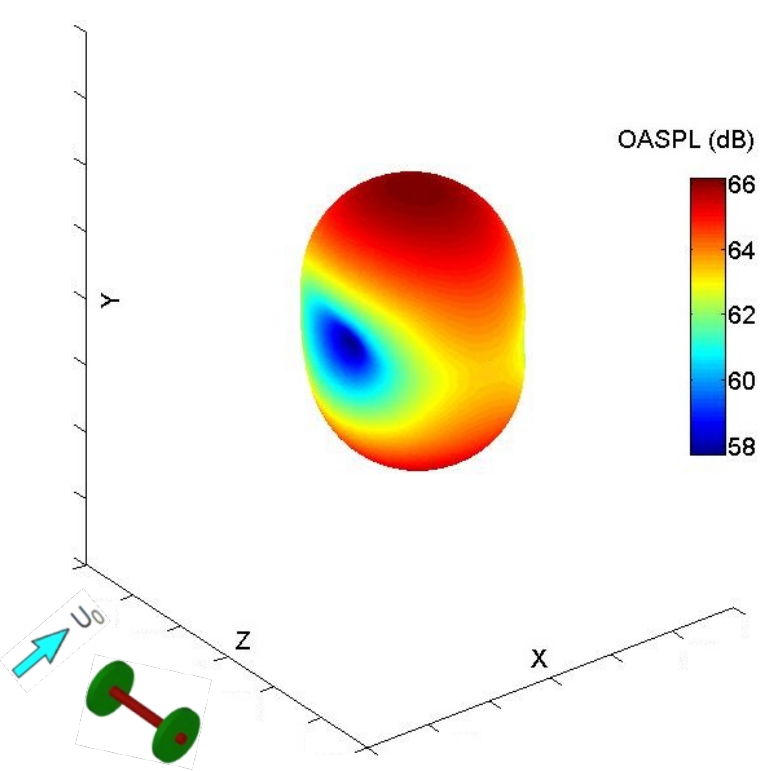

(b) Rear wheelset

Fig. 13. Three-dimensional noise directivity for front and rear wheelsets of simplified bogie

The three-dimensional directivities of noise calculated from the surface source on the front and rear wheelsets of the whole bogie are shown in Figs. 13(a) and 13(b) respectively. The noise levels (starting at $50 \mathrm{~dB}$ ) are represented as the radial distance from the front or rear wheelset geometry centre. It is noted that a distinct dipole pattern of directivity perpendicular to both the freestream direction and the wheelset axis is predicted for the sound radiation from the upstream wheelset (Fig. 13a), indicating that the lift dipole is a dominant noise source. By comparison, the directivity pattern of the downstream wheelset (Fig. 13b) is more uniform in magnitude, resulting from the contributions from the lift and drag dipoles which are aligned perpendicular to each other. This is because the flow separated from the axle of the front wheelset is dominated by the periodic vortex shedding, whereas the rear wheelset is submerged in the upstream wheelset wake and situated in a turbulent condition as shown in Fig. 3, and thus the irregular flow feature makes the noise radiation more broadband and multidirectional. Additionally, it shows that the level of the noise radiated from the rear wheelset is much smaller compared to that from the front wheelset. This is because the turbulent flow convected from the front wheelset is dissipated and the trailing wheelset is subject to a smaller mean incident flow velocity of about $16 \mathrm{~m} / \mathrm{s}$. 


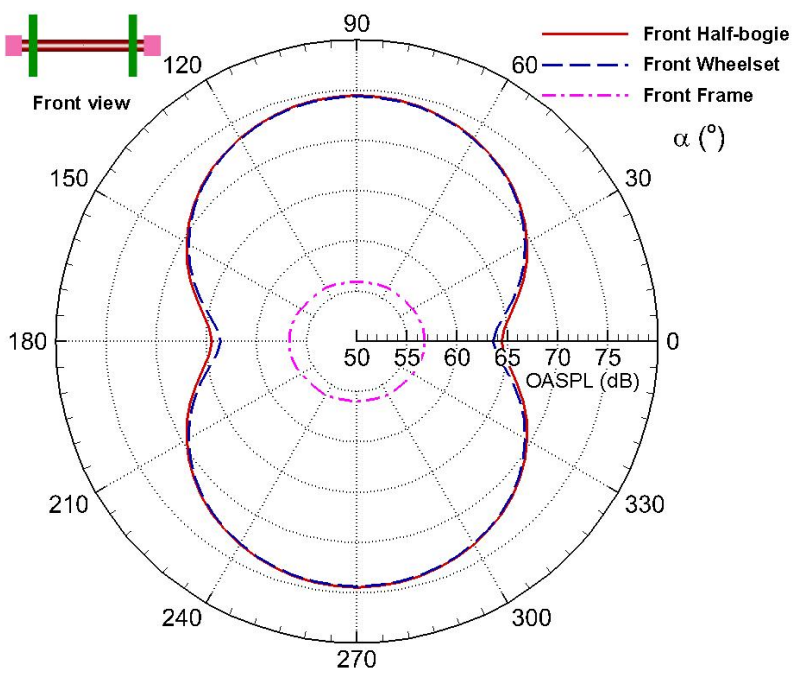

(a) Front half-bogie

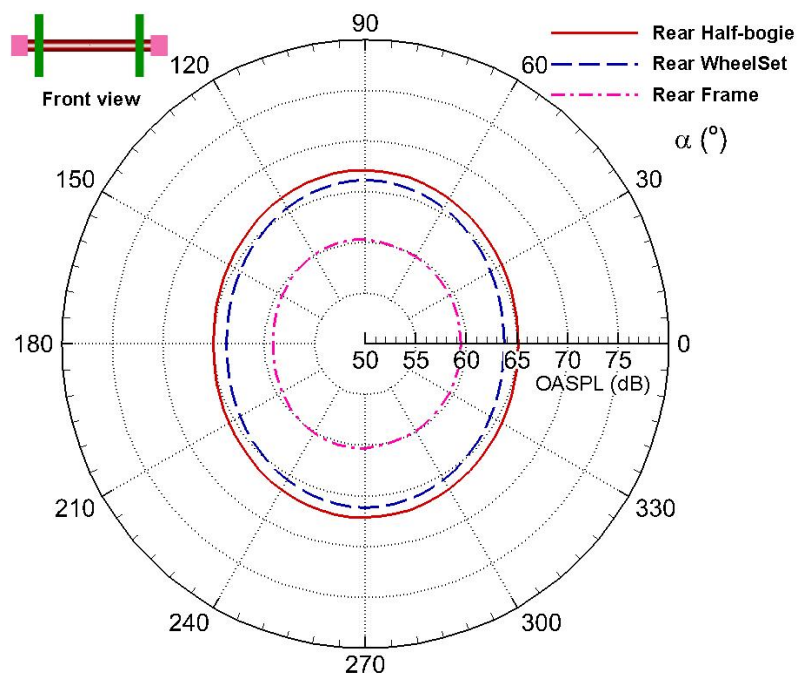

(b) Rear half-bogie

Fig. 14. Noise directivity of front and rear half-bogies in vertical $z-y$ plane

Fig. 14 displays the same results, the noise directivities from the front and rear half parts (divided by the frame transverse mid-plane) of the whole bogie in the vertical $z-y$ plane. In addition, their components are shown. It can be seen that the noise radiated from the rear half-bogie is smaller (up to $7.3 \mathrm{~dB}$ ) than from the front half-bogie. This is because the upstream geometries are in the freestream conditions and the surrounding flow is characterized by large-scale regular vortex shedding; however, the approaching flow around the downstream geometries is highly turbulent but less energetic. Note that the noise is $0.7 \mathrm{~dB}$ higher from the downstream half-bogie at $\alpha=0^{\circ}$ or $180^{\circ}$ along the bogie lateral side, which is due to a slightly stronger flow separation generated from these regions. Moreover, the noise levels for the front half-bogie and front wheelset are very close and the difference in noise level between the rear half-bogie and rear wheelset is around $1 \mathrm{~dB}$. In contrast the noise from the frame is much smaller. This again suggests that for the simplified bogie case, the main noise contributions come from the wheelsets. The noise radiated from the rear frame is $2.5-4.5 \mathrm{~dB}$ larger than from the front frame because of the stronger vortex shedding and flow separation occurring at the frame ends, but is still at least $5 \mathrm{~dB}$ lower than the noise from the corresponding wheelset.

Fig. 15 depicts the three-dimensional directivity pattern (starting at $50 \mathrm{~dB}$ ) of noise radiated from the whole bogie. The noise directivity from the bogie in the vertical $x-y$ plane is compared with that from the tandem wheelsets in Fig. 16. It can be seen that 
for both cases, the directivity pattern exhibits a dipole with an evident radiation bias upwards to the inflow direction and almost symmetrical fore and aft along the horizontal $x-z$ central plane. Note that the similar directivity pattern of sound radiation occurs from the two cases with the slight difference of noise amplitudes between them, which also demonstrates that the wheelsets are the dominant noise sources of the bogie and the noise contribution from the bogie frame is relatively small.

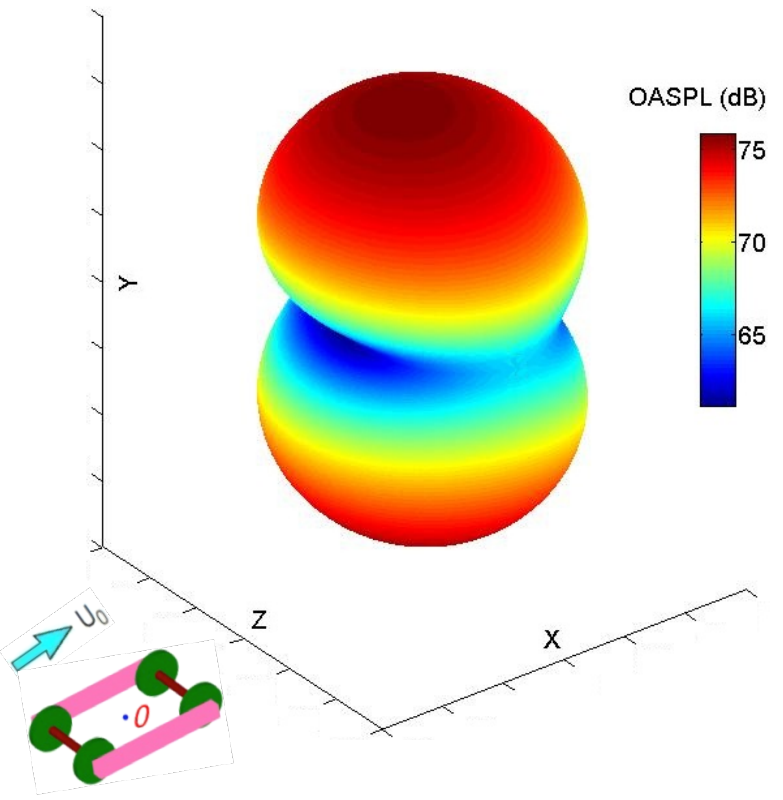

Fig. 15. Three-dimensional noise directivity for simplified bogie

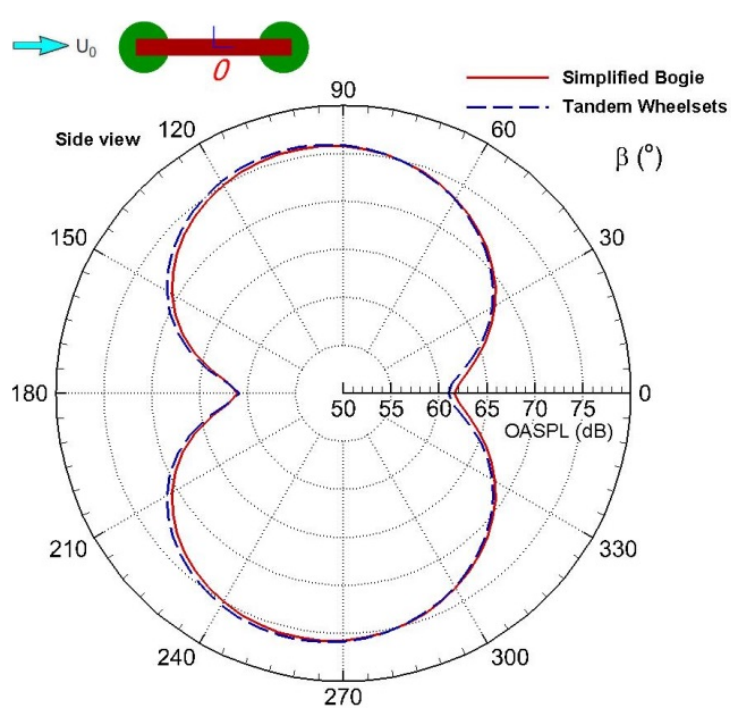

Fig. 16. Noise directivity of whole geometry in vertical $x-y$ plane

\section{Conclusions}

The aerodynamic and aeroacoustic behaviour of the flow past a simplified bogie has been studied using the DDES model and FW-H acoustic analogy. The aerodynamic noise generation and radiation from the tandem wheelsets are also calculated for comparison. It is found that both streamwise and spanwise vortices are generated due to flow separation and vortex shedding around the bogie. The primary behaviour of the flow past the bogie is that the vortices shed from the upstream geometries are convected downstream and impinge on the downstream ones, leading to a highly turbulent wake behind the downstream bodies. For both bogie and tandem-wheelset cases, good agreements are achieved between the numerical predictions and the experimental measurements in terms of the tonal peak characteristics and spectra shapes. The tonal noises are generated with dominant frequencies corresponding to 
the lift dipole due to the vortex shedding around the axles. Furthermore, a vertical dipole pattern of noise radiation is predicted for the upstream wheelset; whereas the downstream wheelset has a multi-directional directivity pattern due to the lift and drag dipoles being aligned perpendicular to each other and its sound generation is relatively weaker. Expressed relative to the geometry centre, the noise directivity has a biased dipole pattern towards the upstream direction as a consequence of large-scale regular vortex shedding produced from the upstream geometries and the downstream bodies are submerged in the decayed and less energetic turbulent incoming flow. Compared to the wheelsets, the frame of the bogie is a minor noise source. These findings are helpful to understand the aerodynamic noise generating mechanisms from the bogie at full scale.

It should be noted that the flow and flow-induced noise behaviour of the bogie inside the bogie cavity with the ground underneath will be different with the isolated bogie case. For a full-scale bogie in reality, the Reynolds number is much higher and the aerodynamic noise characteristics will be changed. The turbulent inflow and the complex geometry will lead to complex flow structures and these will also affect the noise generation. Thus, a compressible flow solver needs to be utilized for flow calculation to consider the acoustic shielding and scattering of sound waves by solid surfaces. Such factors need to be accounted for in future work.

\section{Acknowledgements}

The numerical simulations were performed on the Iridis4 cluster managed by University of Southampton. The first author would like to thank Koen van Mierlo for help in postprocessing of simulated data and Eduardo Latorre Iglesias for the assistance in the experimental measurements. The research studentship provided by University of Southampton is gratefully appreciated.

\section{Appendix A. Grid sensitivity study on the spatial and time resolution in DDES}


A rigorous grid convergence study for complex geometry case is difficult to achieve because of the large unsteady calculations. As a main part of the wheelset, the axle is a typical circular cylinder and therefore a mesh refinement study has been performed on a circular cylinder case and used to provide guidelines for mesh generation. The numerical calculations here have been performed for a cylinder diameter $(D)$ of $20 \mathrm{~mm}$ and freestream velocity $\left(U_{\infty}\right)$ of $64 \mathrm{~m} / \mathrm{s}$ (at a Mach number of 0.19 ), corresponding to experimental data in [21]. In the computational domain, a steady uniform flow is imposed at the upstream inlet; the downstream exit is defined as pressure outlet with a gauge pressure of zero; the top and bottom boundaries are given the conditions of zero-shear slip wall to avoid the need for a high grid density to resolve the wind tunnel boundary layer; the spanwise length of the cylinder is $3 D$ with periodic conditions employed on the two lateral boundaries; a standard no-slip boundary condition is applied for the cylinder surface.

The influence of spatial resolution has been compared by using different grid points in the $x-y$ plane (cases named 'Coarse', 'Baseline' and 'Fine') and the spanwise ( $z$ ) direction; the effect of time resolution has also been examined through reducing the timestep size to one-fifth of that used in the 'Baseline' model. Relative to the 'Baseline' grid, the coarse grids are reduced by a factor of $\sqrt{2}$ in the number of grid points in each ( $x$ and $y$ ) direction and the fine grids are generated by increasing the refinement in each ( $x$ and $y$ ) direction by a factor of 2 . The influence of spatial resolution in the spanwise direction $(z)$ is compared by three different grid sizes of 40,60 and 120 cells with the same $x-y$ plane grids from the 'Baseline' case.
Grid
Grid points $(x, y)$
Grid points (z)
Mesh size
$\Delta t U_{\infty} / D$

\section{Different resolutions in $(x, y)$}

\begin{tabular}{ccccc}
\hline Coarse & 8500 & 60 & $0.51 \mathrm{M}$ & 0.0128 \\
Baseline & 19125 & 60 & $1.15 \mathrm{M}$ & 0.0128 \\
Fine & 76500 & 60 & $4.6 \mathrm{M}$ & 0.0032 \\
\hline \multicolumn{2}{l}{ Different resolutions in $(\mathbf{z})$} & & & \\
\hline Z1 & 19125 & 40 & $0.77 \mathrm{M}$ & 0.0128 \\
Z2 (Baseline) & 19125 & 60 & $1.15 \mathrm{M}$ & 0.0128 \\
Z3 & 19125 & 120 & $1.72 \mathrm{M}$ & 0.0128 \\
\hline
\end{tabular}


Different resolutions in time

\begin{tabular}{ccccc}
\hline T1 (Baseline) & 19125 & 60 & $1.15 \mathrm{M}$ & 0.0128 \\
T2 & 19125 & 60 & $1.15 \mathrm{M}$ & 0.00256 \\
\hline
\end{tabular}

Table 2. Overview of the mesh size and timestep size for grid independence study

The mesh and timestep size for this grid independence study are described in Table 2 where $\Delta t U_{\infty} / D$ represents the non-dimensional timestep. In addition, the influences of mesh and time resolution are summarized in Table 3, the parameters of which are: $y^{+}$ is the dimensionless first-cell spacing, $S t$ the Strouhal number, $C_{L}^{\prime}$ and $C_{D}^{\prime}$ represent root-mean-square fluctuating lift and drag coefficient, $\bar{C}_{D}$ is mean drag coefficient, $\theta_{\text {sep }}$ the separation angle and $L_{r} / D$ the dimensionless recirculation length. It is noted that the lift and drag coefficient exhibit larger modulations for the coarse grid which indicates its inadequate mesh resolution. The fine mesh case has a slightly smaller separation angle, indicating early flow separation and hence resulting in a longer recirculation region behind the cylinder. Furthermore, increasing the grid or time resolution reduces the dominant shedding frequency (seen in terms of $S t$ ) slightly. In general, all the predicted results have been found to exhibit a certain degree of grid convergence. The differences between cases 'Baseline' and 'Fine' are smaller than those between cases 'Coarse' and 'Baseline'. There is little difference in the results between the 'Baseline' case and the case with fine resolution in the $z$ direction. Using a fine timestep also gave little difference in the results.

\begin{tabular}{|c|c|c|c|c|c|c|c|}
\hline Grid & $y^{+}$ & St & $C_{L}^{\prime}$ & $C_{D}^{\prime}$ & $\bar{C}_{D}$ & $\theta_{\text {sep }}$ & $L_{r} / D$ \\
\hline \multicolumn{8}{|c|}{ Different resolutions in $(x, y)$} \\
\hline Coarse & 0.47 & 0.198 & 0.775 & 0.089 & 1.218 & 89.8 & 1.05 \\
\hline Baseline & 0.55 & 0.196 & 0.486 & 0.083 & 1.061 & 86.8 & 1.29 \\
\hline Fine & 0.50 & 0.195 & 0.442 & 0.081 & 1.108 & 85.9 & 1.41 \\
\hline \multicolumn{8}{|c|}{ Different resolutions in $(z)$} \\
\hline Z1 & 0.51 & 0.198 & 0.637 & 0.091 & 1.112 & 88.5 & 1.18 \\
\hline Z2 (Baseline) & 0.55 & 0.196 & 0.486 & 0.083 & 1.061 & 86.8 & 1.29 \\
\hline Z3 & 0.55 & 0.194 & 0.460 & 0.086 & 0.966 & 87.9 & 1.15 \\
\hline \multicolumn{8}{|c|}{ Different resolutions in time } \\
\hline T1 (Baseline) & 0.55 & 0.196 & 0.486 & 0.083 & 1.061 & 86.8 & 1.29 \\
\hline $\mathrm{T} 2$ & 0.49 & 0.195 & 0.493 & 0.087 & 1.124 & 87.8 & 1.10 \\
\hline
\end{tabular}


Table 3. Summary of computations with different mesh and time resolution

Fig. 17 depicts the spectra comparisons of the radiated noises at the observer between the noise predictions from 'Baseline' mesh case and the experiment measurements in [21] as well as the 'Fine' mesh case. It shows that the frequency of the tonal peaks generated by the periodic vortex shedding is accurately predicted. The receiver located in the simulations coincides with the microphone position from the experiment. A limited range of frequencies from $100 \mathrm{~Hz}$ to $1 \mathrm{kHz}$ is available in the experiment. It is found from Fig. 17(a) that good agreements are obtained between the far-field noise predictions and the experimental measurements, for both the shape of the spectra and the overall levels. Compared with the experimental values, the tonal peak level is slightly higher (about $1.5 \mathrm{~dB}$ ) for the numerical simulation. This trend is possibly connected to that the periodic boundary conditions (corresponding to increased coherent shedding and enhanced spanwise uniformity) applied in the numerical calculation and the finite length as well as the free end used in the experiments result in the slight difference of radiated noise between them. Additionally, Fig. 17(b) shows that the noise spectrum becomes a little higher in most of the frequency range above 200 $\mathrm{Hz}$ and the dominant shedding frequency shifts slightly lower as the grid resolution increased and the timestep decreased. This is because compared to the lower resolution case, more vortex amalgamations and interactions are developed within the wake region close to the cylinder surface in the 'Fine' mesh case. However, on the whole, the noise predictions based on the 'Baseline' and 'Fine' grids matches considerably well despite the fact that in the 'Fine' mesh model, the time signal used for noise calculation starts at an earlier time when the initial transient variation might not be removed fully and the frequency resolution utilized is twice that applied in the 'Baseline' mesh case. Therefore, the 'Baseline' case is demonstrated to have adequate resolution and is used to guide the generation of grids in the bogie case. 


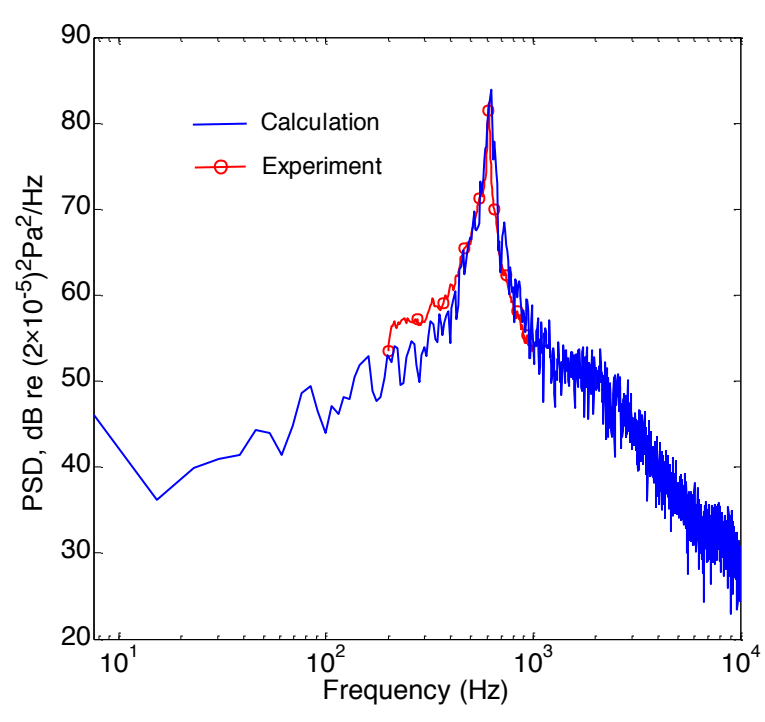

(a) Simulation and experiment

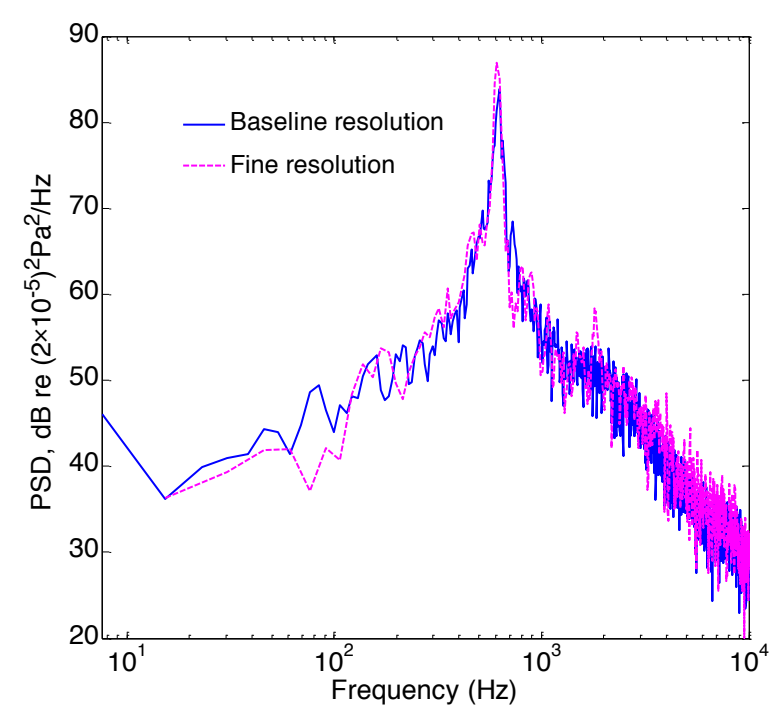

(b) 'Baseline' and 'Fine' grids

Fig. 17. Spectra comparisons of the radiated noise from a circular cylinder case

\section{References}

[1] P.R. Spalart, M.L. Shur, M.Kh. Strelets, A.K. Travin. Initial noise predictions for rudimentary landing gear. Journal of Sound and Vibration, 2011, 330, 4180-4195.

[2] X. Wang, Z.W. Hu, X. Zhang. Aeroacoustic effects of high-lift wing slat track and cut-out system. International Journal of Aeroacoustics, 2013, 12(3): 283-308.

[3] D. P. Lockard. Summary of the tandem cylinder solutions from the benchmark problems for airframe noise computations-I workshop. AIAA Paper 2011-0353, 2011.

[4] G.A. Brès, D. Freed, M. Wessels, S. Noelting, F. Pérot. Flow and noise predictions for the tandem cylinder aeroacoustic benchmark. Phys. Fluids, 2012, 24: 318-337.

[5] D.J. Thompson. Railway noise and vibration: mechanisms, modelling and means of control. Elsevier, Oxford, UK, 2008.

[6] C. Talotte, P.E. Gautier, D.J. Thompson, C. Hanson. Identification, modelling and reduction potential of railway noise sources: a critical survey. Journal of Sound and Vibration, 2003, 267: 447-468.

[7] C.J. Baker. The flow around high speed trains. Journal of Wind Engineering and Industrial Aerodynamics, 2010, 98: 277-298. 
[8] A. Lauterbach, K. Ehrenfried, S. Loose, C. Wagner. Microphone array wind tunnel measurements of Reynolds number effects in high-speed train aeroacoustics. International Journal of Aeroacoustics, 2012, 11: 411-446.

[9] P.E. Gautier, F. Poisson, F. Letourneaux. Noise sources for high speed trains: a review of results in the TGV case. Proceedings of the 9th International Workshop on Railway Noise, Germany, 2007: 71-77.

[10] J.Y. Zhu, Z.W. Hu, D.J. Thompson. Flow simulation and aerodynamic noise prediction for a high-speed train wheelset. International Journal of Aeroacoustics, 2014, 13(7\&8): 533-552.

[11] M. Meskine, F. Pérot, M.S. Kim, D. M. Freed, S. Senthooran, Z. Sugiyama, F. Polidoro, S. Gautier. Community noise prediction of digital high speed train using LBM. AIAA Paper 2013-2015, $19^{\text {th }}$ AIAA/CEAS Aeroacoustics Conference, Germany, 2013.

[12] D.J. Thompson, M.G. Smith, F. Coudret. Application of a component-based approach to modelling the aerodynamic noise from high-speed trains. Proceedings of the 10th International Workshop on Railway Noise, 2010; Notes on Numerical Fluid Mechanics and Multidisciplinary Design, 2012, 118: 427-435.

[13] J.Y. Zhu, Z.W. Hu, D.J. Thompson. Analysis of aerodynamic and aeroacoustic behaviour of a simplified high-speed train bogie. Proceedings of the 11th International Workshop on Railway Noise, 2013; Notes on Numerical Fluid Mechanics and Multidisciplinary Design, 2015, 126: 489-496.

[14] P.R. Spalart, S. Deck, M.L. Shur, K.D. Squires, M.Kh. Strelets, A.K. Travin. A new version of detached-eddy simulation, resistant to ambiguous grid densities. Theoretical and Computational Fluid Dynamics, 2006, 20: 181-195.

[15] J.E. Ffowcs-Williams, D.L. Hawkings. Sound radiation from turbulence and surfaces in arbitrary motion. Philosophical Transactions of the Royal Society of London, 1969, 342: 264-321.

[16] M.J. Lighthill. On sound generation aerodynamically. I. General theory. Proceedings of the Royal Society of London, Series A, Mathematical and Physical Sciences, 1952, 211(1107): 564-587.

[17] K.S. Brentner, F. Farassat. Modelling aerodynamically generated sound of helicopter rotors. Progress in Aerospace Sciences, 2003, 39: 83-120.

[18] M. M. Zdravkovich. Flow around circular cylinders. Vol I: Fundamentals. Oxford University Press, 1997. 
[19] Z.W. Hu, C.L. Morfey, N.D. Sandham. Wall pressure and shear stress spectra from direct numerical simulations of channel flow. AIAA Journal, 2006, 44(7): 1541-1549.

[20] D.J. Moreau, C.J. Doolan. The flow-induced sound of a wall-mounted finite length cylinder with circular and square cross-section. AIAA Paper 2013-2205, $19^{\text {th }}$ AIAA/CEAS Aeroacoustics Conference, Germany, 2013.

[21] W.F. King, E. Pfizenmaier. An experimental study of sound generated by flows around cylinders of different cross-section. Journal of Sound and Vibration, 2009, 328: 318-337. 